\title{
POINTWISE DECAY TO CONTACT DISCONTINUITIES FOR SYSTEMS OF VISCOUS CONSERVATION LAWS*
}

\author{
TAI-PING LIU ${ }^{\dagger}$ AND ZHOUPING XIN ${ }^{\ddagger}$
}

\begin{abstract}
In this paper we study the large time asymptotic behavior toward contact waves for a class of systems of viscous nonlinear conservation laws. Although a given contact discontinuity can not be an asymptotic attractor for the viscous system in general, yet for a large class of systems with some structure constraints, viscous contact waves, which are the exact solutions to the viscous systems and approximate the corresponding contact discontinuities on any finite interval, can be constructed explicitly and are shown to be asymptotically stable with small generic initial perturbations for the viscous systems provided that the strength of the contact discontinuities is suitably small. The leading order large time asymptotic form of the viscous solutions toward a contact wave can be determined a priorily by the distribution of the initial excessive mass, and is a superposition of the viscous contact wave (properly shifted) in the principal linearly degenerate field and nonlinear (or linear) diffusive waves in the transversal fields. The high order deviation of the viscous solutions from its leading order asymptotic ansatz is estimated pointwisely by constructing an approximate fundamental solution of the viscous system linearized around the viscous contact wave, and by analyzing the interactions among viscous contact waves and diffusive waves. The theory is applied to equations for one-dimensional polytropic gases.
\end{abstract}

1. Introduction. We consider the large time asymptotic behavior of the solutions to the initial value problem for the system of viscous conservation laws of the form

$$
\partial_{t} u+\partial_{x} f(u)=\varepsilon \partial_{x}^{2} u, \quad x \in \mathbb{R}^{1}, \quad t>0,
$$

where $u=u(x, t)$, a vector-valued function in $\mathbb{R}^{n}$, represents density variables with $f(u)$ being its corresponding fluxes which is assumed to be smooth, and $\varepsilon>0$ is a viscosity coefficient. (1.1) is supplemented with the initial data

$$
u(x, 0)=u_{0}(x)
$$

satisfying

$$
\lim _{x \rightarrow \pm \infty} u_{0}(x)=u_{ \pm}
$$

where $u_{+}$and $u_{-}$are two constant states in $\mathbb{R}^{n}$.

The qualitative behavior of the solutions to (1.1)-(1.3) is closely related to that of the corresponding inviscid system:

$$
\partial_{t} u+\partial_{x} f(u)=0
$$

It is assumed that (1.4) is hyperbolic, i.e., the Jacobian matrix $f^{\prime}(u)$ has real eigenvalues $\lambda_{1}(u) \leq \lambda_{2}(u) \leq \ldots \leq \lambda_{n}(u)$ with corresponding left and right eigenvectors $l_{i}(u)$ and $r_{i}(u)$ such that

$$
\begin{aligned}
L(u) f^{\prime}(u) R(u) & =\Lambda \equiv \operatorname{diag}\left\{\lambda_{1}(u), \ldots, \lambda_{n}(u)\right\} \\
L(u) R(u) & =I \equiv \text { Identity matrix }
\end{aligned}
$$

*Received February 1, 1997; accepted for publication March 4, 1997.

$\dagger$ Department of Mathematics, Stanford University, Stanford, CA 94305, USA (liu@math.stanford. edu). The research was partially supported by Army Research Grant DAAH04-94-GS0045 and NSF Grant DMS-96-23025.

$\ddagger$ Department of Mathematics, Courant Institute of Mathematical Sciences, New York University, 251 Mercer St., New York, NY 10012, USA (xinz@cims.nyu.edu). The research was partially supported by NSF grants DMS-93-03887, DMS-96-00137, and Department of Energy Grant DeFG02-88ER-25053. 
where $L(u)=\left(\ell_{1}(u), \ldots, \ell_{n}(u)\right)^{t}$ and $R(u)=\left(r_{1}(u), r_{2}(u), \ldots, r_{n}(u)\right)$. An $i$-field is genuinely nonlinear (or linearly degenerate) if $\nabla \lambda_{i}(u) \cdot r_{i}(u) \neq 0\left(\right.$ or $\left.\nabla \lambda_{i}(u) \cdot r_{i}(u) \equiv 0\right)$. A contact discontinuity $[5,10]$, denoted by a triple $\left(u_{l}, u_{r}, s\right)$, is a piecewise constant weak solution to (1.4) such that

$$
f\left(u_{l}\right)-f\left(u_{r}\right)=s\left(u_{l}-u_{r}\right), \quad s=\lambda_{i}\left(u_{l}\right)=\lambda_{i}\left(u_{r}\right)
$$

for some $i, 1 \leq i \leq n$. We will assume that there exists a $p, 1 \leq p \leq n$, such that $\lambda_{p-1}(u)<\lambda_{p}(u)<\lambda_{p+1}(u), p$-field is linearly degenerate, and $\left(u_{+}, u_{-}\right)$forms a contact discontinuity in the $p$-field, i.e.,

$$
f\left(u_{+}\right)-f\left(u_{-}\right)=s\left(u_{+}-u_{-}\right), \quad s=\lambda_{p}\left(u_{+}\right)=\lambda_{p}\left(u_{-}\right) .
$$

By change of the independent variables if necessary, we can assume $s=0$ in (1.6). Let $C_{p}\left(u_{-}\right)$denote the $p$-contact wave curve through $u_{-}$in the state space which is the integral manifold associated with the vector field $r_{p}(u)$. Choosing a nonsingular parameter $\rho$, we can write the $p$-contact wave curve $C_{p}\left(u_{-}\right)$as

$$
C_{p}\left(u_{-}\right)=\left\{u \mid u=u(\rho), \frac{d u}{d \rho}=r_{p}(u(\rho)), u\left(\rho=\rho_{-}\right)=u_{-}\right\}
$$

Then our main structural condition is that both $l_{p}(u)$ and $r_{p}(u)$ are constant along $C_{p}\left(u_{-}\right)$, i.e.,

$$
\nabla r_{p}(u) \cdot r_{p}(u) \equiv 0, \quad \nabla l_{p}(u) \cdot r_{p}(u) \equiv 0 \quad \forall u \in C_{p}\left(u_{-}\right) .
$$

It is noted that the condition (1.8) is trivially verified for the systems of equations for ideal gases (see section 4 ). Now let the initial data $u_{0}(x)$ in (1.2) be a generic small smooth perturbation of the contact discontinuity $\widetilde{U} \equiv\left(u_{-}, u_{+}, 0\right)$ described above. The major questions we will address in this paper are: (1) Is there a global (in time) solution to the Cauchy problem (1.1)-(1.3) for fixed $\varepsilon>0$ ?;

(2) What is the precise large time asymptotic behavior of solutions to (1.1)-(1.3)? More precisely, can $\widetilde{U}=\left(u_{-}, u_{+}, 0\right)$ be an asymptotic state for the viscous solutions to (1.1)-(1.3)? How can one determine the asymptotic (in time) derivation of the viscous solutions from the inviscid contact discontinuity $\tilde{U}$ ?

As observed in [13], an inviscid contact discontinuity can not be an asymptotic attractor for the viscous problem (1.1)-(1.3). It is, in general, only a metastable wave pattern as for the case of polytropic gases [13]. This is in contrast to the nonlinear waves: shock waves and rarefaction waves $[7,9,11,12]$. Indeed, our main results in this paper state, roughly speaking, that although the contact discontinuity $\widetilde{U}$ is not an asymptotic state for the viscous system (1.1) satisfying the structural condition (1.8), yet there exists a smooth exact solution $\bar{U}(x, t)$ to $(1.1)$ which can be constructed explicitly and will be called viscous contact wave. The viscous contact wave has the following desired properties: First, $\bar{U}(x, t)$ approaches the inviscid contact discontinuity $\widetilde{U}$ uniformly on any given finite interval as the viscosity coefficient $\varepsilon$ goes to zero. In fact, the estimate

$$
\|\bar{U}(\cdot, t)-\tilde{U}(\cdot, t)\|_{L^{p}\left(\mathbb{R}^{1}\right)} \leq C(\varepsilon t)^{\frac{1}{2 p}}
$$

holds for a constant $C>0$, and any $p \geq 1$. Second, the viscous contact wave $\bar{U}(x, t)$ is nonlinearly stable for the Cauchy problem (1.1)-(1.3) provided the strength of the 
contact wave, $\left|u_{+}-u_{-}\right|$, is suitably small. Furthermore, the leading order asymptotic structure of the solution to (1.1)-(1.3) is described by the superposition of the viscous contact wave (with center properly shifted) in the $p$-field and nonlinear (or linear) diffusion waves in the transversal fields, and the shift and the masses carried by the diffusion waves can be determined a priori from the distribution of the initial excessive mass. The high order deviation of the solution to (1.1)-(1.3) from its leading order asymptotic ansatz can be estimated pointwisely and optimal rate of convergence (in time) can be obtained.

One of the main difficulties in analyzing contact waves lies in the fact that contact waves are in the linearly degenerate fields and thus less stable [13]. Also since a generic perturbation of a contact wave introduces diffusive waves (see section 2 for details) in the transversal fields, the propagation and interactions of these diffusion waves with the linear contact wave is the other major issue here. Our analysis to overcome these difficulties contains two new elements. The first and most important element is the accurate pointwise estimate on the fundamental solution to the linear parabolic system obtained by linearizing (1.1) around the viscous contact wave, i.e.,

$$
\partial_{t} u+\partial_{x}(\nabla f(\bar{U}(x, t)) u)=\varepsilon \partial_{x}^{2} u
$$

System (1.10) is uniformly parabolic with variable convection coefficients, so the large time pointwise estimate on its fundamental solution matrix poses a nontrivial task. By using characteristic decomposition, we first approximate the fundamental matrix by a diagonal matrix whose $i$-th diagonal element is the fundamental solution to the scalar converted equation

$$
\partial_{t} d+\partial_{x}(a d)=\partial_{x}^{2} d
$$

with $a=a(x, t) \equiv \lambda_{i}(\bar{U}(x, t))$. We then approximate the fundamental solution to the scalar equation (1.11) by the following expression

$$
K_{i}\left(x, t ; x_{0}, t_{0}\right)=\frac{1}{\sqrt{4 \pi\left(t_{0}-t\right)}} \exp \left\{-\frac{m_{i}^{2}\left(x, t ; x_{0}, t_{0}\right)}{4\left(t_{0}-t\right)}\right\}, \quad t<t_{0}
$$

where

$$
\begin{aligned}
m_{p}\left(x, t ; x_{0}, t_{0}\right) & =\left(x-x_{0}\right) \\
m_{i}\left(x, t ; x_{0}, t_{0}\right) & =a(x, t)\left(\int_{x_{0}}^{x} \frac{d y}{a(y, t)}+\left(t_{0}-t\right)\right) \quad i \neq p \\
a(x, t) & \equiv \lambda_{i}(x, t) \equiv \lambda_{i}(\bar{U}(x, t))
\end{aligned}
$$

We will show that this procedure yields the desired pointwise estimate on the fundamental solution to (1.10). Using the fundamental solution matrix to (1.10), one derives (in a standard way) the integral equation for the deviation of the solution to (1.1)-(1.3) from its leading order asymptotic ansatz (the superposition of the shifted viscous contact wave with the diffusion waves in the transversal fields) by Duhamel's principle. Since this integral equation involves also the convolution of the fundamental solution matrix to (1.10) with the sources due to the nonlinear coupling of the diffusion waves in the transversal fields and the nonlinear terms involving the higher order deviation itself, the second main element in our analysis is the study of the interactions among diffusion waves and the viscous contact wave. In particular, the effects on the $p$-th field due to the self interaction of the diffusion waves in the transversal 
directions. This is achieved by describing the precise pointwise asymptotic form for the high order deviation and justifying it through an elaborate iteration processure. This is partly motivated by the work of Liu-Zeng [8] in which the asymptotic stability toward constant state is proved.

There have been intensive studies on the large time asymptotic behavior for solutions to systems of viscous conservation laws including (1.1), see [1,6-8, 10-13]. Almost all the results deal with the nonlinear stability of constants, viscous shock profiles, and rarefaction waves. In particular, pointwise explicit time-asymptotic forms toward constant state for solutions to general quasilinear hyperbolic-parabolic systems of conservation laws have been obtained in [8]. Nonlinear stability of contact discontinuities for the 1-dimensional compressible Euler equation for the polytropic gases with uniform viscosity have been proved in [13] without the rate of convergence. The method employed in [13] is different from our general analysis here which yield optimal rate of convergence.

Several remarks are in order. First, since the system (1.1) has uniform viscosity, so the standard model for compressible Navier-Stokes equations can not fit into this class. However, we do expect that the similar results hold true for the compressible Navier-Stokes equations for the polytropic gases based on the analysis in [13] and this paper. However this is left for the future. On the other hand, it should be noted that the asymptotic behavior toward contact discontinuity for solutions to (1.1)(1.3) is interesting on its own right since (1.1) governs the leading order behavior of many first order (including the Lax-Friedrichs) numerical discretization of the general inviscid system (1.4). Finally, we note that the structural condition (1.8) plays an important role in both our construction of leading order of asymptotic ansatz and the stability analysis. The asymptotic behavior toward contact discontinuity for solutions to general viscous systems without the structural condition (1.8) is an open question.

We conclude this introduction by outlining the rest of this paper. In section 2 , we first construct the leading order asymptotic ansatz as a superposition of a shifted viscous contact wave and nonlinear (or linear) diffusion waves in the transversal fields. The viscous contact wave, which approximates the inviscid contact discontinuity, is constructed by diffusing out the discontinuities along the contact wave curve $C_{p}\left(u_{-}\right)$. The general solution to the Cauchy problem (1.1)-(1.3) can be decomposed as the sum of this leading asymptotic ansatz with a reminder. The error equation for the reminder is obtained. We then state our precise stability theorem. We carry out the pointwise estimates on the fundamental solution matrix to the linear system (1.10) in section 3, where we verify the explicit formulas in (1.12) and (1.13) indeed yields the desired approximation to the fundamental solution matrix of (1.10). We first derive an integral representation formula for the deviation of the solution to (1.1)-(1.3) from its leading order asymptotic ansatz by using the fundamental solution matrix to (1.10) and the Duhamel principle. This is carried out in terms of characteristic variables. Using this representation and the estimate on the fundamental solution matrix, we derive the precise pointwise asymptotic form for the deviation of the solution to (1.1)-(1.3) from the leading order asymptotic ansatz described in section 2 . Finally, in section 4, we show that our general theory applies to the compressible Euler equations for the polytropic gases with uniform viscosity.

2. Asymptotic ansatz and the stability theorem. To describe the leading order large time asymptotic ansatz, we first introduce the viscous contact wave which approximates a given contact discontinuity. 
2.1. Viscous contact wave. Let $\left(u_{-}, u_{+}, s=0\right)$ correspond to a $p$-contact discontinuity so that (1.6) is satisfied with $s=\lambda_{p}\left(u_{+}\right)=\lambda_{p}\left(u_{-}\right)=0$. Then

$$
\tilde{U}(x, t)= \begin{cases}u_{-}, & x<0, t>0 \\ u_{+}, & x>0, t>0\end{cases}
$$

is the contact discontinuity solution to the inviscid system (1.4). Recall that the $p$ contact wave curve through $u_{-}, C_{p}\left(u_{-}\right)$, is defined by (1.7). To define the viscous contact wave, we choose the nonsingular parameter $\rho$ in (1.7) such that

$$
\left\{\begin{array}{l}
\text { (i) } u\left(\rho_{-}\right)=u_{-}, \quad u\left(\rho_{+}\right)=u_{+}, \text {and } \rho_{-}<\rho_{+} ; \\
\text {(ii) }\left\{\begin{array}{l}
\partial_{t} \rho(x, t)=\varepsilon \partial_{x}^{2} \rho(x, t), \\
\rho(x, t=-1)=\rho_{-}+\left(\rho_{+}-\rho_{-}\right) H(x),
\end{array} \quad x \in \mathbb{R}^{1}, t>-1,\right.
\end{array}\right.
$$

here $H(x)$ is the Heaviside function. We can now define the viscous contact wave associated with $\left(u_{-}, u_{+}, s=0\right)$ as follows.

DeFinition 2.1. Let $u(\rho)$ denote the state on the contact wave curve $C_{p}\left(u_{-}\right)$ through $u_{-}$, and let the parameter $\rho$ be chosen as in (2.2). Then the viscous contact wave associated with $\left(u_{-}, u_{+}, s=0\right)$ is defined to be $\bar{U}(x, t) \in C_{p}\left(u_{-}\right)$such that

$$
\bar{U}(x, t)=u(\rho(x, t)) \quad \text { for } \quad x \in \mathbb{R}^{1}, t \geq 0 .
$$

It follows from the definition of $C_{p}\left(u_{-}\right)$that

$$
\partial_{t} \bar{U}(x, t)=r_{p}(\bar{U}) \partial_{t} \rho, \quad \partial_{x} \bar{U}(x, t)=r_{p}(\bar{U}) \partial_{x} \rho
$$

and

$$
\partial_{x}^{2} \bar{U}(x, t)=r_{p}(\bar{U}) \partial_{x}^{2} \rho+\nabla r_{p}(\bar{U})-r_{p}(\bar{U})\left(\partial_{x} \rho\right)^{2}
$$

And so

$$
\begin{aligned}
\partial_{t} \bar{U}+\partial_{x} f(\bar{U})-\varepsilon \partial_{x}^{2} \bar{U}= & \left(\partial_{t} \rho\right) r_{p}(\bar{U})+\lambda_{p}(\bar{U}) r_{p}(\bar{U}) \partial_{x} \rho-\varepsilon r_{p}(\bar{U}) \partial_{x}^{2} \rho \\
& -\varepsilon \nabla r_{p}(\bar{U}) r_{p}(\bar{U})\left(\partial_{x} \rho\right)^{2}=\left(\partial_{t} \rho+\lambda_{p}(\bar{U}) \partial_{x} \rho-\varepsilon \partial_{x}^{2} \rho\right) r_{p}(\bar{U})- \\
& -\varepsilon \nabla r_{p}(\bar{U}) \cdot r_{p}(\bar{U})\left(\partial_{x} \rho\right)^{2}=0
\end{aligned}
$$

where we have used $(2.2)$, the fact $\lambda_{p}(\bar{U}(x, t))=\lambda_{p}\left(u_{-}\right)=s=0$, and the structural condition (1.8). We list some properties of the viscous contact wave in the following lemma which will be used later.

LEMMA 2.2. The viscous contact wave $\bar{U}(x, t)$ in (2.3) is a smooth function for all $(x, t) \in \mathbb{R}^{1} \times \mathbb{R}_{+}^{1}$, and satisfies

(i) $\partial_{t} \bar{U}+\partial_{x} f(\bar{U})-\varepsilon \partial_{x}^{2} \bar{U}=0 \forall(x, t) \in \mathbb{R}^{1} \times \mathbb{R}_{+}^{1}$;

(ii) $\|\bar{U}(\cdot, t)-\widetilde{U}(\cdot, t)\|_{L^{q}} \leq C(\varepsilon(1+t))^{\frac{1}{2 q}}$ for all $q \geq 1$, where $\widetilde{U}(x, t)$ is the inviscid contact discontinuity given in (2.1), and $C$ is a positive constant;

(iii) Set $G(x, t)=\frac{1}{\sqrt{4 \pi(t+1)}} \exp \left\{-\frac{x^{2}}{4(t+1)}\right\}$. Then for all $(x, t) \in \mathbb{R}^{1} \times \mathbb{R}_{+}^{1}, \mid \partial_{x} \bar{U}$ $(x, t)|\leq C \delta| G(x, t) \mid$, and $\left|\partial_{t} \bar{U}(x, t)\right| \leq C \delta \varepsilon\left|\partial_{x} G(x, t)\right|$, where $\delta=\left|u_{+}-u_{-}\right|$ is the strength of the contact wave, and $C$ is an absolute constant.

Thus $\bar{U}(x, t)$ gives the desired viscous approximation to the inviscid contact discontinuity. This completes the construction of viscous contact waves. Next we turn to the diffusion waves. 
2.2. Diffusion waves. To fix the idea, we will concentrate on the case that system (1.4) is strictly hyperbolic so that the wave speeds are strictly separated, and make some remarks on the non-strictly hyperbolic case. To simplify the presentation, we will use the following convention unless otherwise stated. By $l_{i}, r_{i}, \alpha_{i} \equiv \nabla \lambda_{i} r_{i}$, and $\lambda_{i}$, etc., we always mean these quantities evaluated at $u_{-}$for $i<p$ and $u_{+}$for $i>p$, and $l_{p}=l_{p}\left(u_{-}\right)=l_{p}\left(u_{+}\right)$, etc. From now on, we will assume also that $\varepsilon=1$ in (1.1). A generic perturbation of the contact wave should also introduce waves in the transversal characteristic fields. These are the nonlinear (or linear) diffusion waves introduced by Liu [6], which are governed by the converted Burgers equation

$$
\partial_{t} \theta_{i}+\lambda_{i} \partial_{x} \theta_{i}+\partial_{x}\left(\frac{1}{2} \nabla \lambda_{i} r_{i} \theta_{i}^{2}\right)=\partial_{x}^{2} \theta_{i}, \quad i \neq p .
$$

Due to the conservation form of (2.4), the mass carried by the diffusion wave,

$$
m_{i}=\int_{\mathbb{R}^{1}} \theta_{i}(x, t) d x
$$

is a time invariant. By Hopf-Cole transform, the solution to (2.4)-(2.5) can be explicitly written as [6]

$$
\theta_{i}(x, t)=\frac{1}{\sqrt{t+1}} \chi_{i}\left(\frac{x-\lambda_{i}(t+1)}{\sqrt{t+1}}\right), \quad i \neq p
$$

with

$$
\chi_{i}(y)=\frac{\alpha_{i}^{-1}\left(\exp \left\{\alpha_{i} m_{i} / 2\right\}-1\right) \exp \left\{-y^{2}\right\}}{\sqrt{\pi}+\left(\exp \left(\alpha_{i} m / 2\right)-1\right) \int_{y}^{+\infty} \exp \left\{-\xi^{2}\right\} d \xi}
$$

provided that $\alpha_{i} \equiv \nabla \chi_{i} r_{i} \neq 0$, (i.e., the $i$-characteristic field is genuinely nonlinear at $u_{-}(i<p)$ or $\left.u_{+}(i>p)\right)$. In the case of linear degeneracy, $\alpha_{i}=0$, then instead of $(2.7)$, the $\chi_{i}$ in $(2.6)$ is given by

$$
\chi_{i}(y)=\frac{m_{i}}{\sqrt{4 \pi}} \exp \left\{-y^{2}\right\}
$$

We remark that in the case of non-strictly hyperbolic system, we will require that the inviscid system (1.4) has a strictly convex entropy extension ([4]). In this case $\lambda_{i}$ may not be simple. Assume that the multiplicity of $\lambda_{i}$ is $\mu_{i}$. Then the governing equations (2.4) become a system for a $\mu_{i}$-vector $\theta_{i}$. Then by the analysis of Chern [1], the diffusion wave is the unique self-similar solution of the form (2.6) with $\chi_{i}(y=$ $\tilde{\chi}_{i}(y) \exp \left\{-y^{2}\right\}$, where $\tilde{\chi}_{i}$ and all its derivatives are uniformly bounded by $C\left|m_{i}\right|$. Thus the qualitative behavior of the diffusion waves in the non-strictly hyperbolic case is similar to the one for strictly hyperbolic systems.

Finally, we set $\theta(x, t)$ to be the superposition of all the diffusion waves in the transversal fields, i.e.,

$$
\theta(x, t)=\sum_{i \neq p} \theta_{i}(x, t) r_{i}
$$

where the mass $m_{i}$ carried by each diffusion wave is to be determined. This will be done by studying the distribution of the initial excessive masses. 
2.3. Leading order asymptotic ansatz. The leading order time-asymptotic ansatz for the solution to (1.1)-(1.3) will be a superposition of a shift viscous contact wave with diffusion waves in the transversal characteristic fields. So the main task is to determine the center of the viscous contact wave and the masses carried by the diffusions in the transversal fields. To this end, we first observe that

$$
\int_{\mathbb{R}^{1}} \theta(x, t) d x=\sum_{i \neq p}\left(\int_{\mathbb{R}^{1}} \theta_{i}(x, t) d x\right) r_{i}=\sum_{i \neq p} m_{i} r_{i}
$$

and

$$
\begin{aligned}
& \int_{\mathbb{R}^{1}}\left(\bar{U}\left(x+x_{0}, t\right)-\bar{U}(x, t)\right) d x=\int_{\mathbb{R}^{1}}\left(\int_{0}^{x_{0}} \frac{\partial}{\partial y} \bar{U}(x+y, t) d y\right) d x \\
= & \int_{\mathbb{R}^{1}}\left(\int_{0}^{x_{0}} \frac{\partial}{\partial x} \bar{U}(x+y, t) d y\right) d x=\int_{0}^{x_{0}}\left(\int_{\mathbb{R}^{1}} \frac{\partial}{\partial x} \bar{U}(x+y, t) d x\right) d y \\
= & x_{0}\left(u_{+}-u_{-}\right)
\end{aligned}
$$

are time invariant. Next, since for $\delta=\left|u_{+}-u_{-}\right|$small, $u_{+}-u_{-}$is parallel to $r_{p}$ up to leading order, so $\gamma_{1} \ldots, \gamma_{p-1}, u_{+}-u_{-}, \gamma_{p+1}, \ldots, \gamma_{n}$ forms a basis in $\mathbb{R}^{n}$. We thus can decompose the initial excessive mass as follows

$$
\int_{\mathbb{R}^{1}}\left(u_{0}-\bar{U}\right)(x, 0) d x=x_{0}\left(u_{+}-u_{-}\right)+\sum_{i \neq p} m_{i} r_{i} .
$$

It is noted that $(2.12)$ determines $x_{0}, m_{i}(i \neq p)$ uniquely given the initial excessive mass. With $x_{0}, m_{i}(i \neq p)$ so determined, we now define the leading order time asymptotic ansatz for the solution of (1.1)-(1.3) as

$$
u^{a}(x, t)=\bar{U}\left(x+x_{0}, t\right)+\theta(x, t) \equiv \bar{U}\left(x+x_{0}, t\right)+\sum_{i \neq p} \theta_{i}(x, t) r_{i}
$$

It follows from (1.10)-(2.12) that

$$
\begin{aligned}
\int_{\mathbb{R}^{1}}\left(u_{0}(x)-u^{a}(x, 0)\right) d x= & \int_{\mathbb{R}^{1}}\left(u_{0}(x)-\bar{U}(x, 0)\right) d x \\
& +\int_{\mathbb{R}^{1}}\left(\bar{U}(x, 0)-u^{a}(x, 0)\right) d x \\
= & x_{0}\left(u_{+}-u_{-}\right)+\sum_{i \neq p} m_{i} r_{i}+ \\
& +\int_{\mathbb{R}^{1}}\left(\bar{U}(x, 0)-\bar{U}\left(x+x_{0}, 0\right)\right) d x \\
& -\int_{\mathbb{R}^{1}} \theta(x, 0) d x=0 .
\end{aligned}
$$


Due to the conservation laws for the viscous contact wave $\bar{U}(x, t)$, the diffusion wave $\theta(x, t)$, and the solution $u(x, t)$ to (1.1)-(1.3), we have for all $t \geq 0$ that

$$
\int_{\mathbb{R}^{1}}\left(u(x, t)-u^{a}(x, t)\right) d x=0 \quad \forall t>0 .
$$

It follows from Lemma 2.2, (2.4), and some direct computations that

$$
\begin{aligned}
& \partial_{t} u^{a}+\partial_{x}\left(f\left(u^{a}\right)\right)-\partial_{x}^{2} u^{a}= \\
= & \partial_{t} \bar{U}+\partial_{x} f(\bar{U})-\partial_{x}^{2} \bar{U}+\partial_{t} \theta+\partial x\left(f\left(u^{a}\right)-f(\bar{U})\right)-\partial_{x}^{2} \theta \\
= & \partial_{x}\left\{\frac{1}{2} \sum_{i=1}^{n}\left(\sum_{j \neq i, j \neq p} l_{i} \nabla^{2} f(\bar{U})\right)\left(r_{j}, r_{j}\right) \theta_{j}^{2}\right) r_{i}+0(1)|\theta|^{3} \\
& -\sum_{i \neq p}\left[\left(f^{\prime}(\bar{U})-f^{\prime}\left(u_{i}\right)\right) \theta_{i}+\frac{1}{2} \theta_{i}^{2} l_{i}\left(\nabla^{2} f(\bar{U})-\nabla^{2} f\left(u_{i}\right)\right)\left(r_{i}, r_{i}\right)+\right. \\
& \left.+\frac{1}{2} \sum_{\substack{j, k) \neq(i, i) \\
j, k \neq p \\
j \neq k}} l_{i} \nabla^{2} f(\bar{U})\left(\gamma_{k}, r_{j}\right) \theta_{j} \theta_{k}\right] r_{i}+ \\
& \left.+\frac{1}{2}\left(\sum_{\substack{i, j \neq p \\
i \neq j}} l_{p} \nabla^{2} f(\bar{U})\left(r_{i}, r_{j}\right) \theta_{i} \theta_{j}\right) r_{p}\right\} \equiv \partial_{x} \mathcal{E}
\end{aligned}
$$

where $\mathcal{E}$ denote the quantity in the bracket $\{\cdots\}$ in the previous step, and we also used the convention that $u_{i}=u_{-}$for $i<p$ and $u_{i}=u_{+}$for $i>p$, and the simple identity $l_{i}(u) \cdot \nabla^{2} f(u)\left(r_{i}(u), r_{i}(u)\right)=\nabla \lambda_{i}(u) \cdot r_{i}(u)$. Due to the speeds of propagation of waves, one can obtain from the constructions of viscous contact wave and the diffusion waves, see (2.2)-(2.3) and (2.6)-(2.8), that

$$
\left|\bar{U}(x, t)-u_{i}\right|\left|\theta_{i}(x, t)\right|=0(1) \delta\left|m_{i}\right| \exp \{-C(t+|x|)\} \quad \text { for } \quad i \neq p,
$$

and

$$
\left|\theta_{j}(x, t) \theta_{k}(x, t)\right|=0(1)\left|m_{j}\right|\left|m_{k}\right| \exp \{-C(t+|x|)\} \quad \text { for } \quad j \neq k,
$$

for some constant $C>0$. It now follows easily that the error in (2.16) has the following estimate

$$
\begin{aligned}
\mathcal{E}= & \frac{1}{2} \sum_{i=1}^{n} \sum_{\substack{j \neq i \\
j \neq p}}\left(l_{i} \nabla^{2} f(\bar{U})\left(r_{j}, r_{j}\right) \theta_{j}^{2}\right) r_{i}+0(1)|\theta|^{3} \\
& +0(1)\left(\delta m_{0}+m_{0}^{2}\right) \exp \{-C(t+|x|)\},
\end{aligned}
$$

where

$$
m_{0} \equiv \sum_{i=1}^{n}\left|m_{i}\right|
$$

The purpose of the present paper is to show that indeed the derivation, $u(x, t)-$ $u^{a}(x, t)$, of the solution $u(x, t)$ to (1.1)-(1.3) from the function $u^{a}(x, t)$ defined in (2.13) decays faster than $u^{a}(x, t)$ and thus to justify the name of $u^{a}(x, t)$. 
2.4. The stability theorem. With the leading order time asymptotic ansatz $u^{a}(x, t)$ constructed in the previous subsection, our main result in this paper states that there exists a unique global (in time) solution, $u(x, t)$, to the Cauchy problem (1.1)-1.3) in the neighborhood of $u^{a}$, and whose leading order large time asymptotic behavior is completely described by $u^{a}(x, t)$. Furthermore, the deviation, $u(x, t)-$ $u^{a}(x, t)$, of the solution $u(x, t)$ to (1.1)-(1.3) from its leading order time asymptotic ansatz, $u^{a}(x, z)$, can be estimated pointwisely. More precisely we have the following theorem.

Theorem Consider the Cauchy problem (1.1)-(1.3) with $\varepsilon$ being fixed to be one. Suppose that the system (1.1) is either strictly hyperbolic or with convex entropy extension and the p-characteristic field is linearly degenerate. Let $\bar{U}(x, t)$ be the viscous contact wave as defined by (2.3) corresponding to the p-th characteristic field. Let's decompose the initial excessive mass as in (2.12), so the $u^{a}(x, t)$, a superposition of a shifted viscous contact wave with transversal diffusion waves, be constructed as in (2.13). Then there exist suitable small positive constants $\delta_{1}$ and $\delta_{2}$ such that if

$$
\begin{aligned}
& u_{0}-\bar{U} \in H^{1}\left(\mathbb{R}^{1}\right), \\
& \delta \equiv\left|u_{+}-u_{-}\right| \leq \delta_{1}, \\
& \int_{-\infty}^{+\infty}(1+|x|)\left|u_{0}(x)-\bar{U}(x)\right| d x+\int_{-\infty}^{\infty}\left(1+|x|^{2}\right)\left|u_{0}(x)-\bar{U}(x)\right|^{2} d x+ \\
& \|(1+|x|)^{\alpha}\left|u_{0}(x)-\bar{U}(x)\right|_{L^{\infty}} \leq \delta_{2}, \quad \text { for } \quad \alpha>1
\end{aligned}
$$

then there exist a unique smooth global (in time) solution, $u(x, t)$, to the Cauchy problem (1.1)-(1.3) with the following properties:

$$
\begin{aligned}
& \text { (a) } u-\bar{U} \in C\left([0, \infty): H^{1}\left(\mathbb{R}^{1}\right), \quad u-u^{a} \in C\left([0, \infty): H^{1}\left(\mathbb{R}^{1}\right)\right)\right. \\
& \text { (b) } \lim _{t \rightarrow \infty}\left\|u(\cdot, t)-\bar{U}\left(\cdot+x_{0}, t\right)\right\|_{L^{p}\left(\mathbb{R}^{1}\right)}=0 \quad \forall \text { all } p>1, \\
& \quad \text { and }\left\|u(\cdot, t)-\bar{U}\left(\cdot,+x_{0}, t\right)\right\|_{L^{1}\left(\mathbb{R}^{1}\right)} \quad \text { is uniformly bounded in time, } \\
& \text { (c)for all } x \in \mathbb{R}^{1}, \quad t \geq 0,
\end{aligned}
$$

$$
\begin{aligned}
u(x, t)= & u^{a}(x, t)+\partial_{x}\left(\sum_{i=1}^{n} \nu_{i}(x, t) r_{i}(\bar{U}(x, t))\right) \\
\equiv & \bar{U}\left(x+x_{0}, t\right)+\sum_{i \neq p} \theta_{i}(x, t) r_{i}+\sum_{i=1}^{n} \partial_{x} \nu_{i}(x, t) r_{i}(\bar{U}(x, t))+ \\
& +\sum_{i=1}^{n} \nu_{i}(x, t) \nabla r_{i}(\bar{U}(x, t)) \frac{\partial \bar{U}}{\partial_{x}}
\end{aligned}
$$

with

$$
\begin{aligned}
\nu_{i}(x, t) & =0(1)\left(\delta_{1}+\delta_{2}\right)\left(\left|x-\lambda_{i} t\right|^{2}+(t+1)\right)^{-\frac{1}{4}} \\
\partial_{x} \nu_{i}(x, t) & =0(1)\left(\delta_{1}+\delta_{2}\right)\left(\left|x-\lambda_{i} t\right|^{2}+(t+1)\right)^{-\frac{1}{4}}(1+t)^{-\frac{1}{2}}
\end{aligned}
$$

and

$$
\partial_{x} \nu_{i}(x, t)=0(1)\left(\delta_{1}+\delta_{2}\right)|x|^{-\alpha} \quad \text { for } \quad|x| \geq C_{0} t, \quad 1<\alpha<5 / 4
$$


where $C_{0} \geq 4 \max _{i, x, t}\left(\left|\lambda_{i}(\bar{U}(x, t))\right|\right)$ is a positive constant.

Before we move to the proof of the theorem, a few remarks are in order.

(1) The property (a) in the theorem can be obtained by combining a slight modification of the weighted energy estimates announced in [13] with the approximate parametrix analysis, presented in this paper, applying to the linear coupled diffusion waves in [13]. Also decay estimate in $L_{p},(\mathrm{~b})$, in the theorem is a simple consequence of our pointwise estimate (2.23)-(2.26). So our analysis will be concentrated on the property (c) in the theorem.

(2) For given initial data $u_{0}$ in (1.2) satisfying (1.3), the center, $x_{0}$, of the viscous constant wave, and the masses, $m_{i}$, carried by the transversal diffusion waves are uniquely determined by the decomposition of the initial excessive mass (2.12). Thus the leading order asymptotic ansatz, $u^{a}$, is determined a priorily by the initial data. This is so mainly due to our structural condition (1.8). In general, we do not expect that this is the case, and $x_{0}$ has to be determined dynamically. This is, however, left for future.

(3) Though the theorem deals with system (1.1) which is of uniform viscosity. We expect that the analysis in this paper combined with ideas developed by Liu and Zeng in [8] for the propagations of diffusion waves should enable us to generalize the results is this paper to more physical systems, in particular, to the 1-dimensional NavierStokes equations for the polytropic gases. This is currently under consideration.

\section{Stability analysis.}

3.1. Integral error equation and characteristic decomposition. Let $u^{a}(x$, $t$ ) be th leading asymptotic ansatz constructed in $\S 2.3$ (see (2.13)). Without loss of generality, we assume that the amount of shift in the center of viscous contact wave, $x_{0}$, is zero. Suppose now that $u(x, t)$ is a smooth solution to the Cauchy problem (1.1)-(1.3). We can decompose $u(x, t)$ as follows:

$$
u(x, t)=u^{a}(x, t)+\bar{w}(x, t) \equiv \bar{U}(x, t)+\theta(x, t)+\bar{w}(x, t) .
$$

By (1.1) and (2.16), the resulting system governing the deviation, $\bar{w}(x, t)$, of $u(x, t)$ from $u^{a}(x, t)$ becomes

$$
\left\{\begin{array}{l}
\partial_{t} \bar{w}+\partial_{x}\left(f(\bar{U}+\theta+\bar{w})-f\left(u^{a}\right)\right)-\partial_{x}^{2} \bar{w}=-\partial_{x} \mathcal{E} \\
\bar{w}(x, 0)=u_{0}(x)-\bar{U}(x, 0)-\theta(x, 0)
\end{array}\right.
$$

where $\mathcal{E}$ is given in (2.17). And (2.15) gives

$$
\int \bar{w}(x, t) d x=0 \text { for all } t \geq 0
$$

This enables us to write

$$
\bar{w}(x, t)=\frac{\partial}{\partial x} w(x, t)
$$

Substituting (3.3) into (3.2) and integrating the resulting systems with respect to $x$, one has the integrated error equation for $w(x, t)$

$$
\partial_{t} w+f^{\prime}(\bar{U}) \partial_{x} w-\partial_{x}^{2} w=E,
$$




$$
w(x, 0)=w_{0}(x)=\int_{-\infty}^{x}\left(u_{0}(x)-\bar{U}(x, 0)-\theta(x, 0)\right) d x
$$

where

$$
\begin{aligned}
E & \equiv-\mathcal{E}-\left(f\left(u^{a}+\partial_{x} w\right)-f\left(u^{a}\right)-f^{\prime}(\bar{U}) \partial_{x} w\right) \\
& =-\mathcal{E}-\left(f^{\prime \prime}(\bar{U})\left(\theta, \partial_{x} w\right)+\frac{1}{2} f^{\prime \prime}(\bar{U})\left(\partial_{x} w, \partial_{x} w\right)\right)-0(1)\left(|\theta|^{3}+\left|\partial_{x} w\right|^{3}\right)
\end{aligned}
$$

Note that

$$
\begin{aligned}
&\left|\int_{0}^{ \pm \infty}\right| \int_{x}^{ \pm \infty}\left[u_{0}(y)-\bar{U}(y)-\theta(z, 0)\right] d y|d x| \leq 4\left|\int_{0}^{+\infty}\right| u_{0}(y)-\bar{U}(y)-\left.\theta(y, 0)\right|^{2} y^{2} d y \mid, \\
&\left|\int_{0}^{ \pm \infty}\right| \int_{x}^{ \pm \infty}\left[u_{0}(y)-\bar{U}(y)-\theta(y, 0)\right] d y|d x| \leq\left|\int_{0}^{ \pm \infty}\right| u_{0}(y)-\bar{U}(y)-\theta(y, 0)|| y|d y|, \\
&(1+|x|)^{\frac{1}{2}}\left|\int_{x}^{ \pm \infty}\left[u_{0}(y)-\bar{U}(y)-\theta(y, 0)\right] d y\right| \leq \int_{\infty}^{+\infty}(1+|y|)^{2}\left|u_{0}(y)-\bar{U}(y)-\theta(y, 0)\right|^{2} d y
\end{aligned}
$$

which can be verified easily by using the Hölder inequality and change of order of integrations. It follows from the simple inequalities, the explicit forms of the viscous contact wave (Lemma 2.2) and diffusion waves ((2.6)-(2.8)), and the assumptions (2.19)-(2.21) that

$$
\begin{gathered}
w(x, 0)=w_{0}(x) \in H^{2}\left(\mathbb{R}^{1}\right) \cap L^{1}\left(\mathbb{R}^{1}\right) \\
\left\|w_{0}\right\|_{H^{1}\left(\mathbb{R}^{1}\right)}^{2}+\left\|w_{0}\right\|_{L^{1}\left(\mathbb{R}^{1}\right)}+ \\
+\left\|(1+|x|)^{\frac{1}{2}}\left|w_{0}\right|\right\|_{L^{\infty}\left(\mathbb{R}^{1}\right)}+\left\|(1+|x|)^{\alpha}\left|w_{0}^{\prime}\right|\right\|_{L^{\infty}\left(\mathbb{R}^{1}\right)} \leq C \delta_{2}
\end{gathered}
$$

with a fixed positive constant $C$, and $\delta_{2}$ and $\alpha$ are given in the theorem.

We need only to show that the Cauchy problem for (3.4) with initial data (3.5) satisfying (3.7)-(3.8) has a unique global (in time) solution $w(x, t)$ which is of the desired pointwise asymptotic form (2.24)-(2.26). Since (3.4) is a uniform parabolic system, it is standard to obtain the local existence and uniqueness of the smooth solution for the Cauchy problem in a Banach space. The long time existence and asymptotic behavior will follow provided that we can establish some a priori estimates on the solution. The rest of this is devoted to derive such a priori estimates. This will be conveniently done in terms of the characteristic variables

$$
v(x, t)=L(\bar{U}) w(x, t)
$$

where $L(\bar{U})$ is the left eigenvector matrix associated with $f^{\prime}(\bar{U})$ (see Definition 1.5). So one has

$$
w(x, t)=\sum_{i=1}^{n} v_{i}(x, t) r_{i}(\bar{U}(x, t))
$$


and system (3.4) becomes

$$
\partial_{t} v+\Lambda \partial_{x} v-\partial_{x}^{2} v=e_{1}+e_{2}
$$

where

$$
\begin{aligned}
\Lambda= & \operatorname{diag}\left\{\lambda_{1}(\bar{U}), \lambda_{2}(\bar{U}), \ldots, \lambda_{n}(\bar{U})\right\} \\
e_{1}= & \left(\partial_{t} L(\bar{U})+\Lambda \partial_{x} L(\bar{U})-\partial_{x}^{2} L(\bar{U})\right) R(\bar{U}) v+2\left(L(\bar{U}) \partial_{x} R(\bar{U})\right) \partial_{x} v \\
& -2 \partial_{x} L(\bar{U}) \partial_{x} R(\bar{U}) v \\
e_{2}= & L(\bar{U}) E
\end{aligned}
$$

The goal now is to derive the pointwise asymptotic forms (2.24)-(2.26) for the solution $v(x, t)$ to (3.11). The main advantage of the system (3.11) for the characteristic variable $v(x, t)$ over (3.4) is that for weak waves, the coupling of waves in different characteristic fields is weak so that the fundamental solution matrix for the linearized equations for (3.11) can be well approximated by a diagonal matrix whose diagonal elements are the approximated scalar dual waves corresponding to the left hand side of (3.11).

We conclude this section by list some simple estimates on the right hand side of (3.11) which will be useful for our later analysis.

LEMMA 3.1. The coupling and nonlinear terms in (3.11) admit the following estimates

$$
\begin{aligned}
\left(e_{1}+e_{2}\right)_{p}= & 0(1)\left(\theta^{2}+\sum_{i \neq p}\left|\partial_{x} v_{i}\right|^{2}+\sum_{i \neq p}\left|\partial_{x} \rho\right|^{2}\left|v_{i}\right|^{2}+\right. \\
& \left.+|\theta|^{3}+\left|\partial_{x} w\right|^{3}+\left(\delta m_{0}+m_{0}^{2}\right) \exp \{-C(t+|x|)\}\right)
\end{aligned}
$$

for $i \neq p,\left(e_{1}\right)_{i}=0(1)\left(\sum_{j \neq p}\left|\partial_{x} \rho\right|\left(\left|v_{j}\right|\left(1+\left|\partial_{x} \rho\right|\right)+\left|\partial_{x} v_{j}\right|\right)\right)$,

$$
\text { for } \begin{aligned}
i \neq p,\left(e_{2}\right)_{i}= & 0(1)\left(\sum_{j \neq i} \theta_{j}^{2}+|\theta|\left|\partial_{x} v\right|+\sum_{j \neq p}|\theta|\left|\partial_{x} \rho\right|\left|v_{j}\right|\right. \\
& \left.+\sum_{j \neq p}\left|\partial_{x} v_{j}\right|^{2}+\sum_{j \neq p}\left|\partial_{x} v_{p}\right|\left(\left|\partial_{x} v_{j}\right|+\mid \partial_{x} \rho\right)\left|v_{j}\right|\right) \\
& +\sum_{j \neq p}\left|\partial_{x} \rho\right|^{2}\left|v_{j}\right|^{2}+ \\
& +|\theta|^{3}+\left|\partial_{x} w\right|^{3}+\left(\delta m_{0}+m_{0}^{2}\right) \exp \{-C(t+|x|)\} .
\end{aligned}
$$

Proof of Lemma 3.1. This lemma follows from direct computations by using the structure of viscous contact wave and transversal diffusion waves and the structural conditions (1.8). Indeed, direct calculation gives that for all $i=1,2, \ldots, n$

$$
\begin{aligned}
\left(e_{1}\right)_{i}= & \sum_{j} v_{j} l_{i}(\bar{U}) \nabla r_{j}(\bar{U}) \cdot r_{p}(\bar{U})\left(\partial_{t} \rho+\lambda_{i}(\bar{U}) \partial_{x} \rho-\partial_{x}^{2} \rho\right) \\
& -\sum_{j}\left[\left(\partial_{x} \rho\right)\left(\partial_{x} v_{j}\right)\left(l_{i}(\bar{U}) \nabla r_{j}(\bar{U}) \cdot r_{p}(\bar{U})\right)+\right.
\end{aligned}
$$




$$
\begin{aligned}
& \left.+\left(\partial_{x} \rho\right)^{2} v_{j} l_{i}(\bar{U})\left(\nabla\left(\nabla r_{j}(\bar{U}) \cdot r_{p}(\bar{U})\right) \cdot r_{p}(\bar{U})\right)\right] \\
= & \sum_{j} \lambda_{i}(\bar{U})\left(\partial_{x} \rho\right) v_{j}\left(l_{i}(\bar{U}) \nabla r_{j}(\bar{U}) \cdot r_{p}(\bar{U})\right)+ \\
& -\sum_{j}\left[\left(\partial_{x} \rho\right) \partial_{x} v_{j} l_{i}(\bar{U})\left(\nabla r_{j}(\bar{U}) \cdot r_{p}(\bar{U})\right)+\right. \\
& \left.+\left(\partial_{x} \rho\right)^{2} v_{j} l_{i}(\bar{U})\left(\nabla\left(\nabla r_{j}(\bar{U}) \cdot r_{p}(\bar{U})\right) \cdot r_{p}(\bar{U})\right)\right]
\end{aligned}
$$

where we have used the equation for $\rho(x, t)$ in $(2.2)$ in the second inequality above. Note that the identity $\nabla l_{p}(\bar{U}) \cdot r_{p}(\bar{U})=0$ is equivalent to

$$
l_{p}(\bar{U})\left(\nabla r_{j}(\bar{U}) \cdot r_{p}(\bar{U})\right) \equiv 0 \quad \forall j=1,2, \ldots, n,
$$

which is derived by taking directional derivative of the identity $l_{p}(u) \cdot r_{i}(u)=\delta_{p_{i}}$ along the direction $r_{p}(u)$ to get

$$
\left.0=\left(\nabla l_{p}(u) \cdot r_{p}(u)\right) r_{(} u\right)+l_{p}(u)\left(\nabla r_{j}(u) \cdot r_{p}(u)\right), \quad j=1,2, \ldots, n .
$$

Differentiating (3.17) with respect $x$ and using the structure of the viscous contact wave, $\bar{U}(x, t)$, one gets

$\left(\nabla l_{p}(\bar{U}) \cdot r_{p}(\bar{U})\right)\left(\nabla r_{j}(\bar{U}) \cdot r_{p}(\bar{U})\right)+l_{p}(\bar{U})\left[\nabla\left(\nabla r_{j}(\bar{U}) \cdot r_{p}(\bar{U})\right) \cdot r_{p}(\bar{U})\right]=0, \quad j=1,2, \ldots, n$.

And so

$$
l_{p}(\bar{U})\left[\nabla\left(\nabla r_{j}(\bar{U}) \cdot r_{p}(\bar{U})\right) \cdot r_{p}(\bar{U})\right]=0, \quad j=1,2, \ldots n .
$$

It follows from (1.8) and (3.16)-(3.18) that

$$
\begin{aligned}
\left(e_{1}\right)_{p} \equiv 0 & \\
\left(e_{1}\right)_{i}= & \sum_{j \neq p} \lambda_{i}(\bar{U})\left(l_{i}(\bar{U}) \nabla r_{j}(\bar{U}) \cdot r_{p}(\bar{U})\right)\left(\partial_{x} \rho\right) v_{j}- \\
& -\sum_{j \neq p}\left[\left(\partial_{x} \rho\right)\left(\partial_{x} v_{j}\right) l_{i}(\bar{U})\left(\nabla r_{p}(\bar{U}) \cdot r_{p}(\bar{U})\right)+\right. \\
& \left.+\left(\partial_{x} \rho\right)^{2} v_{j} l_{i}(\bar{U})\left(\nabla\left(\nabla r_{j}(\bar{U}) \cdot r_{p}(\bar{U})\right) \cdot r_{p}(\bar{U})\right)\right] \\
= & 0(1)\left(\sum_{j \neq p}\left|\partial_{x} \rho\right|\left(\left|v_{j}\right|\left(1+\left|\partial_{x} \rho\right|\right)+\left|\partial_{x} v_{j}\right|\right)\right) \text { for } i \neq p
\end{aligned}
$$

which is (3.14). Next, one has from (3.6) and (3.12) that

$$
\begin{aligned}
\left(e_{2}\right)_{p}=l_{p}(\bar{U}) E=- & l_{p}(\bar{U}) f^{\prime \prime}(\bar{U})\left(\theta, \partial_{x} w\right) \\
& -\frac{1}{2} l_{p}(\bar{U}) f^{\prime \prime}(\bar{U})\left(\partial_{x} w, \partial_{x} w\right)-l_{p}(\bar{U}) \mathcal{E}+0(1)\left(|\theta|^{3}+\left|\partial_{x} w\right|^{3}\right) .
\end{aligned}
$$

To do this computation, one notes that

(3.21) $l_{p}(\bar{U}) \nabla^{2} f(\bar{U})\left(r_{i}(\bar{U}), r_{j}(\bar{U})\right)=\lambda_{i}(\bar{U}) l_{p}(\bar{U})\left(\nabla r_{i}(\bar{U}) \cdot r_{j}(\bar{U})\right)$ for all $i$ and $j$,

due to the assumption $\lambda_{p}(\bar{U})=\lambda_{p}\left(u_{+}\right)=\lambda_{p}\left(u_{-}\right)=0$, and

$$
l_{p}(\bar{U}) \nabla^{2} f(\bar{U})\left(r_{p}(\bar{U}), r_{i}(\bar{U})\right)=0 \quad \text { for } \quad i=1,2, \ldots, n,
$$


which follows from (3.17) and the known identity

$$
\begin{aligned}
l_{p}(u) \nabla^{2} f(u)\left(r_{p}(u), r_{i}(u)\right)=\nabla & \lambda_{i}(u) \cdot r_{p}(u) l_{p}(u) \cdot r_{i}(u) \\
& +\left(\lambda_{i}(u)-\lambda_{p}(u)\right) l_{p}(u) \nabla r_{i}(u) \cdot r_{p}(u) .
\end{aligned}
$$

By using (1.8), (3.17)-(3.18), (3.21)-(3.22), and $w_{t}=R(\bar{U}) \partial_{x} v+\partial_{x}(R(\bar{U})) v$, one can calculate that

$$
\begin{aligned}
& l_{p}(\bar{U}) \nabla^{2} f(\bar{U})\left(\theta, \partial_{x} w\right) \\
& =l_{p}(\bar{U}) \nabla^{2} f(\bar{U})\left(\sum_{i \neq p} \theta_{i} r_{i}(\bar{U}), R(\bar{U}) \partial_{x} v\right)+ \\
& +l_{p}(\bar{U}) \nabla^{2} f(\bar{U})\left(\sum_{i \neq p} \theta_{i} r_{i}(\bar{U}), \partial_{x}(R(\bar{U})) v\right)+ \\
& +l_{p}(\bar{U}) \nabla^{2} f(\bar{U})\left(\sum_{j \neq p} \theta_{j}\left(r_{j}-r_{j}(\bar{U}), \partial_{x} w\right)\right. \\
& =\sum_{\substack{i \neq p \\
j \neq p}}\left(\lambda_{i}(\bar{U}) l_{p}(\bar{U})\left(\nabla r_{i}(\bar{U}) \cdot r_{j}(\bar{U})\right) \theta_{i} \partial_{x} v_{j}+\right. \\
& +0(1)\left(|v|+\left|\partial_{x} w\right|\right)\left(m_{0} \delta\right) \exp \{-C(t+|x|)\}, \\
& \frac{1}{2} l_{p}(\bar{U}) \nabla^{2} f(\bar{U})\left(\partial_{x} w, \partial_{x} w\right) \\
& =\frac{1}{2} \sum_{i \neq p, j \neq p}\left(\partial_{x} v_{i}\right)\left(\partial_{x} v_{j}\right)\left(\lambda_{i}(\bar{U}) l_{p}(\bar{U})\left(\nabla r_{i}(\bar{U}) r_{j}(\bar{U})\right)+\sum_{i \neq p, k \neq p}\left(\partial_{x} v_{i}\right)\right. \\
& \left(\sum_{j \neq p}\left(\partial_{x} \rho\right) v_{j} l_{k}(\bar{U})\left(\nabla r_{j}(\bar{U}) \cdot r_{p}(\bar{U})\right) \cdot \lambda_{i}(\bar{U}) \lambda_{p}(\bar{U})\left(\nabla r_{i}(\bar{U}) \cdot \gamma_{k}(\bar{U})\right)\right)+ \\
& +\frac{1}{2} \sum_{i \neq p, k \neq p}\left(\sum_{j \neq p}\left(\partial_{x} \rho\right) v_{j} l_{k}(\bar{U})\left(\nabla r_{j}(\bar{U}) \cdot r_{p}(\bar{U})\right)\right) \\
& \left(\sum_{j \neq p}\left(\partial_{x} \rho\right) v_{j} l_{i}(\bar{U})\left(\nabla r_{j}(\bar{U}) \cdot r_{p}(\bar{U})\right) \lambda_{k}(\bar{U}) l_{p}(\bar{U})\left(\nabla \gamma_{k}(\bar{U}) \cdot r_{i}(\bar{U})\right),\right.
\end{aligned}
$$

and

$$
l_{p}(\bar{U}) \mathcal{E}=l_{p} \mathcal{E}=\frac{1}{2} \sum_{i \neq p} l_{p} \nabla^{2} f\left(r_{i}, r_{i}\right) \theta_{i}^{2}+0(1)|\theta|^{3}+0(1)\left(\delta m_{0}+m_{0}^{2}\right) \exp \{-C(t+|x|)\} .
$$

This combined with (3.19) yields (3.13) immediately. Since similar calculation gives (3.15), the proof of Lemma 3.1 is complete.

3.2. Approximate scalar dual and an integral representation. We now begin our proof of the theorem by analyzing the asymptotic behavior of solution to (3.11). As it is well-known, the accurate pointwise behavior of solutions to parabolic system is achieved by using the parametrix method. However, the classical treatment of parametrix methods for parabolic systems with variable coefficients deals only with 
local in time estimates [3] except the recent studies on nonlinear waves (see $[6,11,12$, $8,13])$.

Since the linearized form of equation (3.11) has coefficients depending the leading order asymptotic ansatz $u^{a}(x, t)$ which varies in both space and time, so it is difficult to obtain the exact form of the Green's function for the linearized equations. By using the hyperbolic structure and properties of the viscous contact wave, we can approximate this Green's function by approximate scalar dual waves corresponding only to the decoupled scalar operators on the left hand side of (3.11). This approach makes crucial use of the structure of the underlying viscous contact wave and the smallness assumptions (2.20)-(2.21).

Now that the linear operator on the left hand side of (3.11) is diagonal, and each component has a dual of the form

$$
-\partial_{t} \bar{\eta}_{i}-\partial_{x}\left(\lambda_{i}(\bar{U}) \bar{\eta}_{i}\right)-\partial_{x}^{2} \bar{\eta}_{i}=0, \quad j=1,2, \ldots, n .
$$

Thus the fundamental solution matrix to the linear operator $\partial_{t} v+\Lambda \partial_{x} v-\partial_{x}^{2} v$ is a diagonal matrix with $(\mathrm{i}, \mathrm{i})$-component given by $\bar{\eta}_{i}(x, t ; y, T)$ which solves $(3.23)$ on $\mathbb{R}^{1} \times(0, T)$ with data

$$
\bar{\eta}_{i}(x, t=T ; y ; t)=\delta(x-y)
$$

for any given $T>0$ and $y \in \mathbb{R}^{1}$. In the case $i=p, \lambda_{p}(\bar{U}(x, 0))=\lambda_{p}\left(u_{+}\right)=\lambda_{p}\left(u_{-}\right)=$ 0 , so the solution to (3.23)-(3.24) is given uniquely by the heat kernel

$$
\bar{\eta}_{p}(x, t ; T, y)=\frac{1}{\sqrt{4 \pi(T-t)}} \exp \left\{-\frac{(x-y)^{2}}{4(T-t)}\right\} \text {. }
$$

For $i \neq p$, we do not have an explicit solution to (3.23)-(3.24) in general. We thus define an approximate scalar dual $\eta_{i}(x, t ; T, y)$ as follows:

$$
\eta_{i}(x, t ; T, y)=\frac{1}{\sqrt{4 \pi(T-t)}} \exp \left\{-\frac{\left(m_{i}(x, t ; y, T)\right)^{2}}{4(T-t)}\right\} \quad \text { for } \quad x \in \mathbb{R}^{1}, y \in \mathbb{R}^{1}
$$

and $t<T$, where

$$
\begin{aligned}
& m_{p}(x, t ; y, T)=(x-y) \\
& m_{i}(x, t ; y, T)=\lambda_{i}(x, t)\left(\int_{y}^{x} \frac{d \xi}{\lambda_{i}(\xi, t)}+(T-t)\right), \quad i \neq p .
\end{aligned}
$$

Here and from now on, we use the notation $\lambda_{i}(x, t) \equiv \lambda_{i}(\bar{U}(x, t))$. It follows that $\eta_{p}(x, t ; y, T) \equiv \bar{\eta}_{p}(x, t ; y, T)$, and so solves $(3.23)_{p}$ exactly. Furthermore, direct computations show that

$$
\eta_{i}(x, t=T, y, T)=\delta(x-y), \quad i=1,2, \ldots, n,
$$

and

$$
\partial_{t} \eta_{i}+\partial_{x}\left(\lambda_{i}(x, t) \eta_{i}\right)+\partial_{x}^{2} \eta_{i}=E_{i}, \quad i \neq p
$$

$$
\begin{aligned}
E_{i}= & \left(\partial_{x} \lambda_{i}(x, t)\right) \eta_{i} \\
& -\eta_{i}\left[\frac{1}{2}\left(\partial_{x} \lambda_{i}+\frac{\left(\partial_{x} \lambda_{i}\right)^{2}}{\lambda_{i}^{2}}\left(1-\frac{1}{2} \frac{m_{i}^{2}}{T-t}\right)+\frac{\partial_{t} \lambda_{i}+\partial_{x}^{2} \lambda_{i}}{\lambda_{i}}\right) \frac{m_{i}^{2}}{T-t}\right. \\
& \left.+\left(\frac{\partial_{x} \lambda_{i}}{\lambda_{i}}\left(1-\frac{1}{2} \frac{m_{i}^{2}}{T-t}\right)+\frac{1}{2} \frac{\partial_{x} \lambda_{i}}{\lambda_{i}}+\frac{1}{2} \lambda_{i} \partial_{t} n_{i}\right) \frac{m_{i}}{T-t}\right],
\end{aligned}
$$


where $\lambda_{i}=\lambda_{i}(x, t), m_{i}=m_{i}(x, t ; y, T)$, and $n_{i}=n_{i}(x, t ; y)=\int_{y}^{x} \frac{d \xi}{\lambda_{i}(\xi, t)}$.

Taking the scalar product of (3.11) with $\eta=\left(\eta_{1}, \ldots, \eta_{n}\right)$ and integrating the resulting equation we obtain the following integral representation for the solution to (3.11) by using (3.28)--(3.30) and Lemma 3.1:

$$
\begin{aligned}
& v_{p}(y, T)=\int v_{p}(x, 0) \eta_{p}(x, t=0 ; y, T) d x+ \\
& +\int_{0}^{T} \int\left(e_{1}+e_{2}\right)_{p}(x, t) \eta_{p}(x, t ; y, T) d x d t \\
& \equiv \int v_{p}(x, 0) \eta_{p}(x, t=0 ; y, T) d x+ \\
& +\int_{0}^{T} \int 0(1)\left(\theta^{2}+\sum_{i \neq p}\left(\left|\partial_{x} v_{i}\right|^{2}+\left|\partial_{x} \rho\right|^{2}\left|v_{i}\right|^{2}\right)+\left|\partial_{x} w\right|^{3}\right) \\
& (x, t) \eta_{p}(x, t ; y, T) d x d t+ \\
& +\int_{0}^{T} \int 0(1)\left(\delta m_{0}+m_{0}^{2}\right) \exp \{-C(t+|x|)\} \eta_{p}(x, t ; y, T) d x d t \\
& v_{i}(y, T) \\
& =\int v_{i}(x, 0) \eta_{i}(x, t=0 ; y, T) d x+\int_{0}^{T} \int E_{i}(x, t ; y, T) v_{i}(x, t) d x d t+ \\
& +\int_{0}^{T} \int\left(e_{1}+e_{2}\right)_{i}(x, t) \eta_{i}(x, t ; y, T) d x d t \\
& =\int v_{i}(x, 0) \eta_{i}(x, 0 ; y, T) d x+\int_{0}^{T} \int E_{i}(x, t ; y, T) v_{i}(x, t) d x d t+ \\
& +\int_{0}^{T} \int 0(1)\left(\sum_{j \neq p}\left|\partial_{x} \rho\right|\left(\left|v_{j}\right|\left(1+\left|\partial_{x} \rho\right|\right)+\left|\partial_{x} v_{j}\right|\right)\right) \\
& (x, t) \eta_{i}(x, t ; y, T) d x d t+ \\
& +\int_{0}^{T} \int 0(1)\left(\sum_{j \neq i}\left|\theta_{j}\right|^{2}+|\theta|\left|\partial_{x} v\right|+\sum_{j \neq p}\left|\partial_{x} v_{j}\right|^{2}\right. \\
& +\sum_{j \neq p}\left|\partial_{x} v_{p}\right|\left(\left|\partial_{x} v_{j}\right|+\left|\partial_{x} \rho\right|\left|v_{j}\right|\right)+ \\
& \left.+\sum_{j \neq p}\left|\partial_{x} \rho\right|^{2}\left|v_{j}\right|^{2}+|\theta|^{3}+\left|\partial_{x} w\right|^{3}\right)(x, t) \eta_{i}(x, t ; y, T) d x d t+ \\
& +\int_{0}^{T} \int 0(1)\left(\delta m_{0}+m_{0}^{2}\right) \exp \{-C(t+|x|)\} \eta_{i}(x, t ; y, T) d x d t, \quad i \neq p .
\end{aligned}
$$

Here and from now on, by the space integral $\int d x$ we always mean the $\int_{\mathbb{R}^{1}} d x$ unless otherwise stated.

We also need to estimate the derivatives of $v(x, t)$. Thus differentiating the expressions (3.31) with respect to $y$ we obtain

$$
\partial_{y} v_{p}(y, T)=\int v_{p}(x, 0) \partial_{y} \eta_{p}(x, 0 ; y, T) d x+
$$




$$
\begin{aligned}
& +\int_{0}^{T} \int\left(e_{1}+e_{2}\right)_{p}(x, t) \partial_{y} \eta_{p}(x, t ; y, T) d x d t \\
\partial_{y} v_{i}(y, T)= & \int v_{i}(x, 0) \partial_{y} \eta_{i}(x, 0 ; y, T) d x \\
& +\int_{0}^{T} \int \partial_{y} E_{i}(x, t ; y, T) v_{i}(x, t) d x d t+ \\
& +\int_{0}^{T} \int\left(e_{1}+e_{2}\right)_{i}(x, t) \partial_{y} \eta_{i}(x, t ; y, T) d x d t
\end{aligned}
$$

The pointwise estimates on the solution (3.11) will be based on the representation formulas (3.31)-(3.32). This clearly depends on the accuracy of our scalar approximate dual $\eta_{i}(x, t ; y, T)$. Thus before moving to the heavy estimates, we first collect some simple properties concerning the approximate scalar dual waves which will be useful in our later analysis. The first concerns the estimate on the error term $E_{i}$ in (3.29)-(3.30).

Lemma 3.2. For $i \neq p$, let $E_{i}$ be given by (3.30).

(1) There exists positive constant $\mu>0$ such that

$$
\begin{aligned}
E_{i}(x, t ; y, T)= & 0(1) \tilde{\eta}_{i}(x, t ; y, T)\left[\left|\partial_{x} \lambda_{i}(x, t)\right|\left(1+(T-t)^{-1 / 2}\right)\right. \\
& \left.+\delta(T-t)^{-1 / 2}(1+t)^{-\frac{1}{2}}\right]
\end{aligned}
$$

with

$$
\widetilde{\eta}_{i}(x, t ; y, T)=\frac{1}{\sqrt{4 \pi(T-t)}} \exp \left\{-\frac{m_{i}^{2}(x, t ; y, T)}{4 \mu(T-t)}\right\}
$$

(2) Set

$$
\partial_{y} E_{i}(x, t ; y, T)=-\frac{\lambda_{i}(x, t)}{\lambda_{i}(y, t)} \partial_{x} E_{i}(x, t ; y, T)+\widetilde{E}_{i}
$$

Then

$$
\begin{aligned}
\widetilde{E}_{i}= & 0(1) \tilde{\eta}_{i}(x, t ; y, T)\left[| \partial _ { x } \lambda _ { i } ( x , t ) | \left(\left|\partial_{x} \lambda_{i}(x, t)\right|\left(1+(T-t)^{-1 / 2}\right)\right.\right. \\
& \left.\left.+\delta(T-t)^{-1 / 2}(1+t)^{-1 / 2}\right)+\left|\partial_{x}^{2} \lambda_{i}(x, t)\right|\left(1+(T-t)^{-1 / 2}\right)\right]
\end{aligned}
$$

where $\tilde{\eta}_{i}(x, t ; y, T)$ is defined by (3.34) with a suitable positive constant $\mu$.

Proof. We first note that (3.33) follows from the expression (3.30), Lemma 2.2, and the simple estimate

$$
\begin{aligned}
\partial_{t} n(x, t ; y) & =\int_{y}^{x} \frac{-\partial_{t} \lambda_{i}(x, t)}{\lambda_{i}^{2}(\xi, z)} d \xi=-\int_{y}^{x} \frac{\nabla \lambda_{i}(\xi, t) \cdot r_{p}(\xi, t)}{\lambda_{i}^{2}(\xi, t)} \rho_{t}(\xi, t) d \xi \\
& =-\int_{y}^{x} \frac{\nabla \lambda_{i}(\xi, t) \cdot r_{p}(\xi, t)}{\lambda_{i}^{2}(\xi, t)} \partial_{x}^{2} \rho(\xi, t) d \xi=0(1) \delta(1+1)^{-1 / 2}
\end{aligned}
$$

Thus we need only to show (3.35)-(3.36). To this end, we set

$$
E_{i}(x, t ; y, T)=\eta_{i}(x, t ; y, T) h,
$$


so that

$$
\begin{aligned}
& h(x, t ; y, T)=\partial_{x} \lambda_{i}(x, t) \\
& -\left[\frac{1}{2}\left(\partial_{x} \lambda_{i}+\frac{\left(\partial_{x} \lambda_{i}\right)^{2}}{\lambda_{i}^{2}}\left(1-\frac{1}{2} \frac{m_{i}^{2}}{T-t}\right)+\frac{\partial_{t} \lambda_{i}+\partial_{x}^{2} \lambda_{i}}{\lambda_{i}}\right) \frac{m_{i}^{2}}{T-t}+\right. \\
& \left.+\left(\frac{\partial_{x} \lambda_{i}}{\lambda_{i}}\left(1-\frac{1}{2} \frac{m_{i}^{2}}{T-t}\right)+\frac{1}{2} \frac{\partial_{x} \lambda_{i}}{\lambda_{i}}+\frac{1}{2} \lambda_{i} \partial_{t} n_{i}\right) \frac{m_{i}}{T-t}\right] \\
\equiv & \hat{h}\left(x, t, T, m_{i}(x, t ; y, T)\right) .
\end{aligned}
$$

Since

$$
\partial_{y} \eta_{i}=-\frac{\lambda_{i}(x, t)}{\lambda_{i}(y, t)}\left(\partial_{x} \eta_{i}+\frac{\partial_{x} \lambda_{i}(x, t)}{\lambda_{i}(x, t)}\left(\frac{m_{i}^{2}}{2(T-t)}\right) \eta_{i}\right)
$$

one has

$$
\begin{aligned}
\partial_{y} E_{i} & =\left(\partial_{y} \eta_{i}\right) h+\eta_{i}\left(\partial_{y} h\right) \\
& =-\frac{\lambda_{i}(x, t)}{\lambda_{i}(y, t)} \partial_{x} \eta_{i} h-\frac{\lambda_{i}(x, t)}{\lambda_{i}(y, t)}\left(\frac{\partial_{x} \lambda_{i}(x, t)}{\lambda_{i}(x, t)}\right) E_{i}+\eta_{i} \partial_{y} h .
\end{aligned}
$$

On the other hand, it follows from (3.38) that

$$
\begin{aligned}
\partial_{x} h & =\partial_{x} \hat{h}+\partial_{m_{i}} \hat{h} \frac{\partial m_{i}}{\partial x}=\partial_{x} \hat{h}+\partial_{m_{i}} \hat{h}\left(1+\frac{\partial_{x} \lambda_{i}(x, t)}{\lambda_{i}(x, t)}\right) \\
\partial_{y} h & =\partial_{m_{i}} \hat{h} \frac{\partial m_{i}}{\partial y}=-\frac{\lambda_{i}(x, t)}{\lambda_{i}(y, t)} \partial_{m_{i}} \hat{h} \\
& =\frac{\lambda_{i}(x, t)}{\lambda_{i}(y, t)}\left(\partial_{x} \hat{h}+\left(\partial_{m_{i}} \hat{h}\right) \frac{\partial_{x} \lambda_{i}(x, t)}{\lambda_{i}(x, t)} m_{i}-\partial_{x} h\right) .
\end{aligned}
$$

This leads to

$$
\partial_{y} E_{i}=-\frac{\lambda_{i}(x, t)}{\lambda_{i}(y, t)} \partial_{x} E_{i}+\widetilde{E}_{i}
$$

with

(3.40) $\widetilde{E}_{i}=-\frac{\partial_{x} \lambda_{i}(x, t)}{\lambda_{i}(y, t)} \cdot\left(\frac{m_{i}^{2}}{2(T-t)}\right) E_{i}+\frac{\lambda_{i}(x, t)}{\lambda_{i}(y, t)} \eta_{i}\left(\partial_{x} \hat{h}+\frac{\partial_{x} \lambda_{i}(x, t)}{\lambda_{i}(x, t)}\left(\partial_{m_{i}} \hat{h}\right) m_{i}\right)$.

Since

$$
\begin{aligned}
\left(\partial_{m_{i}} \hat{h}\right) m_{i}= & -\left\{\left[\partial_{x} \lambda_{i}+\frac{\left(\partial_{x} \lambda_{i}\right)^{2}}{\lambda_{i}^{2}}\left(1-\frac{1}{2} \frac{m_{i}^{2}}{T-t}\right)+\frac{\partial_{t} \lambda_{i}+\partial_{x}^{2} \lambda_{i}}{\lambda_{i}}\right] \frac{m_{i}^{2}}{T-t}\right. \\
& -\frac{1}{2} \frac{\left(\partial_{x} \lambda_{i}\right)^{2}}{\lambda_{i}^{2}} \frac{m_{i}^{4}}{(T-t)^{2}}-\frac{\partial_{x} \lambda_{i}}{\lambda_{i}} \frac{m_{i}^{2}}{T-t} \frac{m_{i}}{T-t}+ \\
& \left.+\left(\frac{\partial_{x} \lambda_{i}}{\lambda_{i}}\left(1-\frac{m_{i}^{2}}{2(T-t)}\right)+\frac{1}{2} \frac{\partial_{x} \lambda_{i}}{\lambda_{i}}+\frac{1}{2} \lambda_{i} \partial_{t} n_{i}\right) \frac{m_{i}}{T-t}\right\}, \\
\partial_{x} \hat{h}= & -\left\{\frac{1}{2}\left[\partial_{x}^{2} \lambda_{i}+\partial_{x}\left(\frac{\left(\partial \lambda_{i}\right)^{2}}{\lambda_{i}^{2}}\right)\left(1-\frac{1}{2} \frac{m_{i}^{2}}{T-t}\right)+\partial_{x}\left(\frac{\partial_{t} \lambda_{i}+\partial_{x}^{2} \lambda_{i}}{\lambda_{i}}\right)\right] \frac{m_{i}^{2}}{T-t}\right. \\
& \left.+\left[\partial_{x}\left(\frac{\partial_{x} \lambda_{i}}{\lambda_{i}}\right)\left(1-\frac{1}{2} \frac{m_{i}^{2}}{T-t}\right)+\frac{1}{2} \partial_{x}\left(\frac{\partial_{x} \lambda_{i}}{\lambda_{i}}\right)+\frac{1}{2}\left(\lambda_{i} \partial_{t} n_{i}\right)_{x}\right] \frac{m_{i}}{T-t}\right\},
\end{aligned}
$$


and

$$
\partial_{t x}^{2} n_{i}=-\frac{\partial_{t} \lambda_{i}}{\lambda_{i}^{2}}=-\frac{\nabla \lambda_{i}(x, t) \cdot r_{p}(x, t) \partial_{x}^{2} \rho}{\lambda_{i}^{2}}
$$

one can conclude (3.36) by inspecting each terms in (3.40) and using (3.33)-(3.34). Thus Lemma 3.2 is proved.

Next we study some important properties, such that the essential support and effective propagation speed, of the approximate transversal scalar dual waves $\eta_{i}(i \neq p)$ defined by (3.26)-(3.27). Due to the explicit form of $\eta_{i}$, its behavior is determined by analyzing the stationary points of the phase $m_{i}(x, t ; y, T)$. For simplicity, we will only state and prove the results for $\eta_{i}, i>p$, since the complementary case, $i<p$, is similar.

Lemma 3.3. Assume $i>p$. Let $m_{i}(x, t ; y, T)$ be defined by (3.27). Then the following properties hold.

(1) For any fixed $y$ and $T>0$, there exists a unique smooth curve $x=\bar{x}(t ; y, T)$ such that

$$
\begin{aligned}
& \bar{x}(t=T ; y, T)=y, \quad m_{i}(\bar{x}(t ; y, T), t ; y, T)=0, \text { for } t \geq 0, \text { and } \\
& \bar{x}(t ; y, T)=y-\lambda_{i}\left(x_{1}, t\right)(T-t)
\end{aligned}
$$

for some $x_{1}=x_{1}(t ; y, T)$.

(2) There exist positive constants $\mu_{1}$ and $\mu_{2}$ such that

$$
\mu_{1}(x-\bar{x}(t ; y, T))^{2} \leq m_{i}^{2}(x, t ; y, T) \leq \mu_{2}(x-\bar{x}(t ; y, T))^{2} .
$$

(3) For fixed $y$ and $T>0$, there is a unique $\bar{t} \geq 0$ such that

$$
\bar{x}(t ; y, T)= \begin{cases}\geq 0 & t \geq \bar{t} \\ <0 & 0 \leq t<\bar{t} .\end{cases}
$$

(4) Denote by $\lambda_{i}^{+}$the value $\lambda_{i}\left(v_{r}\right)=\lambda_{i}(\bar{U}(\infty, t))$. Then

$$
\left|\bar{x}(t ; y, T)-\left(y-\lambda_{i}^{+}(T-t)\right)\right|=0(1) \delta \sqrt{1+T}, \quad t \geq \bar{t} .
$$

(5) There is a constant $C>0$ such that

a) if $y \geq \lambda_{i}^{+} T+C \sqrt{1+T}$, then $\bar{t} \equiv 0$, and

b) if $0 \leq y \leq \lambda_{i}^{+} T+C \sqrt{1+T}$, and $\bar{t}>0$, then

$$
\bar{t}=\frac{1}{\lambda_{i}^{+}}\left|y-\lambda_{i}^{+} T\right|+0(1) \sqrt{1+T}, \quad \text { and }
$$

$$
(\bar{x}(t ; y, T))^{2} \geq \mu_{3}(t-\bar{t})^{2} \quad \text { for some positive constant } \mu_{3}>0
$$

Proof. Since the proof of this lemma is elementary, we will only sketch it. By assumption that $i>p$, so $\lambda_{i}(x, t)>0$. Thus $m_{i}(x, t ; y, T)=0$ if and only if $H(x, t ; y, T) \equiv \eta_{i}(x, t ; y)+(T-t)=0$. Since $H(x=y, t=T ; y, T)=0$ and $\frac{\partial H}{\partial x}=\partial_{x} n_{i}(x, t ; y)=\frac{1}{\lambda_{i}(x, t)}$ which is bounded and uniformly bounded away from zero, so there exists unique smooth curve $x=\bar{x}(t ; y, T)$ so that

$$
H(\bar{x}(t ; y, T), t ; y, T)=\int_{y}^{\bar{x}(t ; y, T)} \frac{d \xi}{\lambda_{i}(\xi, t)}+(T-t)=0
$$


and (3.41)-(3.42) follow easily. Differentiating (3.47) with respect to $t$ gives

$$
\begin{aligned}
\frac{\partial \bar{x}}{\partial t}(t ; y, T) & =\left(1+\int_{y}^{\bar{x}} \frac{\partial_{t} \lambda_{i}(\xi, t)}{\lambda_{i}^{2}(\xi, t)} d \xi\right) \lambda_{i}(\bar{x}, t) \\
& =\left(1+0(1) \delta(1+t)^{-1 / 2}\right) \lambda_{i}(\bar{x}, t) .
\end{aligned}
$$

Since $\delta$ is assumed to be small, so (3.43) and (3.46) follow. Next, by definition, one has $\bar{x}(t ; y, T) \geq 0$ for $t \geq \bar{t}$. Setting

$$
\bar{x}=y-\lambda_{i}^{+}(T-t)
$$

we obtain from (3.47) that

$$
\bar{x}(t ; y, T)-\bar{x}=\int_{y}^{\bar{x}(t ; y, T)} \frac{\lambda_{i}(\xi, t)-\lambda_{i}^{+}}{\lambda_{i}(\xi, t)} d \xi,
$$

which yields (3.44) since $y \geq \bar{x}(t ; y, T) \geq 0$ and $\int_{0}^{+\infty}\left|\lambda_{i}(\xi, t)-\lambda_{i}^{+}\right| d \xi=0(1) \delta(1+t)^{\frac{1}{2}}$. Clearly (3.44) implies a) of (5). If $\bar{t}>0$, then $\bar{x}(\bar{t} ; y, T)=0$, and so (3.50) becomes

$$
-\bar{x}(\bar{t}, y, T)=\int_{y}^{0} \frac{\lambda_{i}(\xi, \bar{t})-\lambda_{i}^{+}}{\lambda_{i}(\xi, \bar{t})} d \xi,
$$

which gives (3.45) immediately. The proof of Lemma 3.3 is complete.

For simplicity of presentation, we will use the following notations:

$$
\begin{aligned}
d_{i}(x, t) & =\left(\left(x-\lambda_{i}(t+1)\right)^{2}+(t+1)\right)^{-1 / 4}, \\
K(x, t) & =\left(|x|^{2 \alpha-1} \chi(x, t)+(t+1)\right)^{-\frac{1}{2}}, \\
H_{i}(x, t) & =K\left(\left(x-\lambda_{i}(t+1)\right), t\right),
\end{aligned}
$$

where $\chi(x, t)$ is a smooth function which is one for $|x| \geq C_{0}(t+1)$ and zero for $|x| \leq \frac{1}{2} C_{0}(t+1)$. Set also

$$
M(t)=\sup _{0 \leq \tau \leq t} \max _{1 \leq i \leq n}\left\{\left\|v_{i}(\cdot, \tau) d_{i}^{-1}(\cdot, \tau)\right\|_{L^{\infty}}+\left\|\partial_{x} v_{i}(\cdot, \tau) d_{i}^{-1}(\cdot, \tau) H_{i}^{-1}(\cdot, \tau)\right\|_{L^{\infty}}\right\}
$$

Thus on the time interval the solution exists, one has that

$$
\begin{aligned}
\left|v_{i}(x, t)\right| & \leq M(t) d_{i}(x, t), \\
\left|\partial_{x} v_{i}(x, t)\right| & \leq M(t) d_{i}(x, t) H_{i}(x, t),
\end{aligned}
$$

for all $x \in \mathbb{R}^{1}, i=1,2, \ldots, n$. We first note that $M(0) \leq C\left(\delta_{1}+\delta_{2}\right)$ by (3.8). As long as one can obtain a priori bound on $M(t)$ independent of the time, the (3.53) combined with the standard continuity argument yields the global (in time) solution and the desired pointwise asymptotic form (2.24)-(2.26). The technical estimates for the bound on $M(t)$ are derived in the next two sections.

3.3. A priori estimate $I$ - on principal waves. We are now in the position to derive a priori estimate on the solution to system (3.11). We start with the estimate on the waves in the principal direction, $v_{p}(x, t)$ and $\partial_{x} v_{p}(x, t)$. This case is somewhat easier than the ones for the transversal waves due to the fact that the scalar dual $\eta_{p}$ is exact. 
It follows from $(3.31)_{p}$ and (3.53) that for any $y \in \mathbb{R}^{1}, T>0$

$$
\begin{aligned}
\left|v_{p}(y, T)\right| \leq & \int\left|v_{p}(x, 0)\right|\left|\eta_{p}(x, 0 ; y, T)\right| d x+ \\
& +\int_{0}^{T} \int 0(1)|\theta|^{2}(x, t)\left|\eta_{p}(x, t ; y, T)\right| d x d t+ \\
& +0(1) M^{2}(T) \int_{0}^{T} \int \sum_{i \neq p} d_{i}^{2}(x, t) H_{i}^{2}(x, t) \eta_{p}(x, t ; y, T) d x d t+ \\
& +0(1) M^{2}(T) \int_{0}^{T} \int \sum_{i \neq p}\left(\left|\partial_{x} \rho\right|^{2} d_{i}^{2}\right)(x, t) \mid \eta_{p}(x, t ; y, T) d x d t+ \\
& +0(1) M^{3}(T) \int_{0}^{T} \int \sum_{i}\left(\left|\partial_{x} \rho\right|^{3} d_{i}^{3}\right)(x, t) \eta_{p}(x, t ; y, T) d x d t+ \\
& +0(1) M^{3}(T) \int_{0}^{T} \int H_{i}^{3}(x, t) d_{i}^{3}(x, t) \eta_{p}(x, t ; y, T) d x d t \\
& +0(1)\left(\delta m_{0}+m_{0}^{2}\right) \int_{0}^{T} \int \exp \left\{-C(t+|x|) \eta_{p}(x, t ; y, T) d x d t\right. \\
& \equiv \sum_{i=1}^{7} I_{i},
\end{aligned}
$$

with the obvious notations for $I_{i}$. We now proceed to estimate each term above. First,

$$
I_{1}=\int\left|v_{p}(x, 0)\right| \eta_{p}(x, 0 ; y, T) d x \leq\left\|v_{p}(\cdot, 0)\right\|_{L^{1}}\left\|\eta_{p}(\cdot, 0, y, T)\right\|_{L^{\infty}} \leq \frac{C \delta_{2}}{\sqrt{4 \pi T}}
$$

If $y \geq C \sqrt{1+T}(y \leq-C \sqrt{1+T}$ is similar $)$, then

$$
\begin{aligned}
I_{1}= & \int_{-\infty}^{y / 2}\left|v_{p}(x, 0)\right| \frac{1}{\sqrt{4 \pi T}} \exp \left\{-\frac{(x-y)^{2}}{4 T}\right\} d x+ \\
& +\int_{y / 2}^{\infty}\left|v_{p}(x, 0)\right| \eta_{p}(x, 0 ; y, t) d x \\
\leq & \frac{1}{\sqrt{4 \pi T}} \exp \left\{-\frac{y^{2}}{16 T}\right\}\left\|v_{p}(\cdot, 0)\right\|_{L^{2}}+ \\
& +\left\|v_{p}(\cdot, 0)(1+|\cdot|)^{1 / 2}\right\|_{L^{\infty}} \int_{y / 2}^{+\infty} \frac{1}{(1+|x|)^{1 / 2}} \eta_{p}(x, 0 ; g, T) d x \\
\leq & C \delta_{2} \frac{1}{\sqrt{4 \pi T}} \exp \left\{-\frac{y^{2}}{16 T}\right\}+\frac{C \delta_{2}}{(1+|y|)^{\frac{1}{2}}} .
\end{aligned}
$$

And so

$$
d_{p}^{-1}(y, T) I_{1}(y, T) \leq C \delta_{2} \quad \text { for all } y \in \mathbb{R}^{1}, T>0 .
$$

Next, we consider

$$
I_{2}=0(1) \int_{0}^{T} \int|\theta|^{2}(x, t) \eta_{p}(x, t ; y, T) d x d t
$$




$$
\begin{aligned}
& \equiv 0(1) \sum_{i \neq p} \int_{0}^{T} \int \theta_{i}^{2}(x, t) \eta_{p}(x, t ; y, T) d x d t \\
& \leq 0(1) m_{0}^{2} \int_{0}^{T} \frac{1}{\sqrt{T(t+1)}} \exp \left\{-\frac{\left(y-\lambda_{i}(t+1)\right)^{2}}{2 T}\right\} d t
\end{aligned}
$$

where we have used the simple formula

$$
\begin{aligned}
& \exp \left\{-\frac{\left(x-\lambda_{i}(t+1)\right)^{2}}{4 \mu_{1}(t+1)}\right\} \cdot \exp \left\{-\frac{\left(x-y+\lambda_{j}(T-t)^{2}\right.}{\left.4 \mu_{2}(T-t)\right)}\right] \\
= & \exp \left\{-\frac{\mu_{1}(t+1)+\mu_{2}(T-t)}{4 \mu_{1} \mu_{2}(t+1)(T-t)}\left(x-\lambda_{i}(t+1)\right.\right. \\
& \left.\left.-\frac{\mu_{1}(t+1)}{\mu_{1}(t+1)+\mu_{2}(T-t)}\left(y-\lambda_{i}(t+1)-\lambda_{j}(T-t)\right)\right)^{2}\right\} \\
& \exp \left\{-\frac{\left(y-\lambda_{i}(t+1)-\lambda_{j}(T-t)\right)^{2}}{4\left[\mu_{1}(T+1)+\mu_{2}(T-t)\right]}\right\}
\end{aligned}
$$

with any given positive constants $\mu_{1}$ and $\mu_{2}$. We will treat the case $i>p$ only (the complementary case $i<p$ is similar). For $|y| \leq C \sqrt{T+1}$, one has

$$
\begin{aligned}
& \int_{0}^{T} \frac{1}{\sqrt{T(t+1)}} \exp \left\{-\frac{\left(y-\lambda_{i}(t+1)\right)^{2}}{2 T}\right\} d t \\
\leq & 0(1) \int_{0}^{T} \frac{1}{\sqrt{T(t+1)}} \exp \left\{-\frac{C(t+1)^{2}}{T}\right\} d t \leq \frac{0(1)}{T^{1 / 4}} .
\end{aligned}
$$

For $y<-C \sqrt{T+1}$, one has

$$
\begin{aligned}
& \int_{0}^{T} \frac{1}{\sqrt{T(t+1)}} \exp \left\{-\frac{\left(y-\lambda_{i}(t+1)\right)^{2}}{2 T}\right\} d t \\
\leq & \exp \left\{-\frac{y^{2}}{4 T}\right\} \int_{0}^{T} \frac{1}{\sqrt{T(t+1)}} \exp \left\{-\frac{\lambda_{i}^{2}(t+1)^{2}}{4 T}\right\} \\
\leq & 0(1) T^{-1 / 4} \exp \left\{-\frac{y^{2}}{4 T}\right\} .
\end{aligned}
$$

If $y>C \sqrt{T+1}$, we have

$$
\begin{aligned}
& \int_{0}^{T} \frac{1}{\sqrt{T(t+1)}} \exp \left\{-\frac{\left(y-\lambda_{i}(t+1)\right)^{2}}{2 T}\right\} d t \\
\leq & 0(1) \int_{0}^{T} \frac{1}{\sqrt{T(t+1)}} \exp \left\{-\frac{\left(y-\lambda_{i} t\right)^{2}}{\mu T}\right\} d t \\
\leq & \int_{0}^{\frac{y}{2 \lambda_{i}}} \frac{1}{\sqrt{T(t+1)}} \exp \left\{-\frac{\left(y-\lambda_{i} t\right)^{2}}{\mu T}\right\} d t \\
& +\int_{\frac{y}{2 \lambda_{i}}}^{T} \frac{1}{\sqrt{T(t+1)}} \exp \left\{-\frac{\left(y-\lambda_{i}(t+1)\right)^{2}}{\mu T}\right\} d t \\
\leq & 0(1) T^{-1 / 4} \exp \left\{-\frac{y^{2}}{4 \mu T}\right\}+0(1)|y|^{-1 / 2} .
\end{aligned}
$$


It follows from (3.59)-(3.61) that

$$
d_{p}^{-1}(y, T) I_{2}(y, T) \leq C m_{0}^{2} \leq C \delta_{2}^{2}
$$

Direct calculation shows that

$$
\begin{aligned}
I_{7} & \equiv 0(1)\left(\delta m_{0}+m_{0}^{2}\right) \int_{0}^{T} \int \exp \{-C(t+|x|)\} \eta_{p}(x, t ; y, T) d x d t \\
(3.63) & \leq \begin{cases}0(1)\left(\delta m_{0}+m_{0}^{2}\right)(1+T)^{-1 / 2} & \text { if }|y| \leq \sqrt{T+1} \\
0(1)\left(\delta m_{0}+m_{0}^{2}\right)\left(T^{-\frac{1}{2}} \exp \left\{-\frac{y^{2}}{\mu T}\right\}+\exp \left\{-C_{1}|y|\right\}\right), & \text { otherwise. }\end{cases}
\end{aligned}
$$

Thus

$$
d_{p}^{-1}(y, T) I_{7}(y, T) \leq 0(1)\left(\delta m_{0}+m_{0}^{2}\right) \leq C\left(\delta_{1}^{2}+\delta_{2}^{2}\right) .
$$

We now turn to the integrals involving nonlinear terms. First, by (3.58) and Lemma 2.2 we can obtain that

$$
\begin{aligned}
(3.65) I_{4} \equiv & 0(1) M^{2}(T) \int_{0}^{T} \int \sum_{i \neq p}\left(\left|\partial_{x} \rho\right|^{2} d_{i}^{2}\right)(x, t) \eta_{p}(x, t ; y, T) d x d t \\
\leq & 0(1) M^{2}(T) \exp \left\{-\frac{y^{2}}{2 T}\right\} \sum_{i \neq p} \int_{0}^{T} \int \frac{1}{(1+t) \sqrt{T-t}} \\
& \exp \left\{-\frac{(t+1)+2(T-t)}{4(t+1)(T-t)}\left(x-\frac{t+1}{(t+1)+2(T-t)} y\right)^{2} d_{i}^{2}(x, t) d x d t\right. \\
\leq & 0(1) M^{2} T^{-1 / 2} \log (1+T) \exp \left\{-\frac{y^{2}}{2 T}\right\}
\end{aligned}
$$

And similarly, one has

$$
\begin{aligned}
I_{5} & \equiv 0(1) M^{3}(T) \int_{0}^{T} \int \sum_{i}\left(\left|\partial_{x} \rho\right|^{3} d_{i}^{3}\right)(x, t) \eta_{p}(x, t ; y, T) d x d t \\
& \leq 0(1) M^{3}(T) T^{-1 / 2} \exp \left\{-\frac{y^{2}}{2 T}\right\}
\end{aligned}
$$

Next, we consider

$$
\begin{aligned}
I_{3} \equiv & 0(1) M^{2}(T) \int_{0}^{T} \int \sum_{i \neq p} d_{i}^{2}(x, t) H_{i}^{2}(x, t) \eta_{p}(x, t ; y, T) d x d t \\
= & 0(1) M^{2}(T) \sum_{i \neq p} \int_{0}^{T} \int_{\left|x-\lambda_{i}(t+1)\right| \leq C \sqrt{t+1}}\left(d_{i}^{2} H_{i}^{2}\right)(x, t) \eta_{p}(x, t ; y, T) d x d t+ \\
& +0(1) M^{2}(T) \sum_{i \neq p} \int_{0}^{T} \int_{\left|x-\lambda_{i}(t+1)\right| \geq C \sqrt{t+1}}\left(d_{i}^{2} H_{i}^{2}\right)(x, t) \eta_{p}(x, t ; y, T) d x d t \\
\equiv & I_{31}+I_{32} .
\end{aligned}
$$

Since

$$
I_{31} \leq 0(1) M^{2}(T) I_{2}(y, T) m_{0}^{-2}
$$


one needs only to estimate $I_{32}$. Note that

$$
\begin{aligned}
(3.68) I_{32} \leq & 0(1) M^{2}(T) \sum_{i \neq p} \int_{0}^{T} \int_{\left|x-\lambda_{i}(t+1)\right| \geq C \sqrt{t+1}} \frac{1}{1+t} d_{i}^{2}(x, t) \eta_{p}(x, t ; y, T) d x d t \\
\leq & 0(1) M^{2}(T) \sum_{i \neq p} \int_{0}^{T} \frac{d t}{1+t}\left(\int_{\left|x-\lambda_{i}(t+1)\right| \geq C \sqrt{t+1}} d_{i}^{4}(x, t) d x\right)^{1 / 2} \\
& \left(\int_{\left|x-\lambda_{i}(t+1)\right| \geq C \sqrt{t+1}} \eta_{p}^{2}(x, t ; y, T) d x\right)^{\frac{1}{2}} \\
\leq & 0(1) M^{2}(T) \sum_{i \neq p} \int_{0}^{T} \frac{1}{(1+t)^{5 / 4}} \frac{1}{(T+t)^{\frac{1}{4}}} d t=0(1) M^{2}(T) T^{-\frac{1}{4}} .
\end{aligned}
$$

It remains to bound $I_{32}$ for $|y| \geq 2 C \sqrt{T+1}$. Rewrite each space integral in $I_{32}$ as

$$
I(t) \equiv \int_{|z| \geq C \sqrt{t+1}} \frac{1}{(t+1)} \frac{1}{\sqrt{T-t}} \exp \left\{-\frac{(z-\bar{y})^{2}}{4(T-t)}\right\} \frac{1}{z} d z
$$

with $\bar{y}=y-\lambda_{i}(t+1)$. Without loss of generality, we assume $i>p$. If $y \leq-2 C \sqrt{T+1}$, then

$$
\begin{aligned}
\int_{0}^{T} I(t) d t \leq & \int_{0}^{T}\left(\int_{-\infty}^{\frac{1}{2} \bar{y}}+\int_{\frac{1}{2} \bar{y}}^{-C \sqrt{t+1}}+\int_{C \sqrt{t+1}}^{+\infty}\right) \\
& \left(\frac{1}{t+1} \frac{1}{\sqrt{T-t}} \exp \left\{-\frac{(z-\bar{y})^{2}}{4(T-t)}\right\} \frac{1}{z} d z d t\right) \\
\leq & \int_{0}^{T} \frac{0(1)}{(1+t)|\bar{y}|} d t\left(\int_{-\infty}^{\frac{1}{2} \bar{y}} \frac{1}{\sqrt{T-t}} \exp \left\{-\frac{(z-\bar{y})^{2}}{4(T-t)}\right\} d z\right)+ \\
& +\int_{0}^{T} \frac{1}{1+t} \frac{1}{\sqrt{T-t}} \exp \left\{-\frac{\bar{y}^{2}}{4 \mu(T-t)}\right\} \\
& \left(\int_{\bar{y} / 2}^{-C \sqrt{t+1}}+\int_{C \sqrt{t+1}}^{+\infty}\right) \exp \left\{-\frac{z^{2}}{4 \mu(T-t)}\right\} \frac{d z}{z} d t \\
\leq & \frac{0(1)}{|y|^{1 / 2}}+0(1) T^{-1 / 4} \exp \left\{-y^{2} / 4 \mu T\right\}
\end{aligned}
$$

We now assume $y>2 C \sqrt{T+1}$. There are two subcases

Case 1. $t \in[0, T] \cap\{t: \bar{y} \leq 2 C \sqrt{t+1}\} \equiv J_{1}$. Then $(t+1) \geq C y$. Thus

$$
\begin{aligned}
I(t) & \leq \frac{1}{1+t}\left(\int_{|z| \geq C \sqrt{t+1}} \frac{1}{T-t} \exp \left\{-\frac{(z-\bar{y})^{2}}{2(T-t)}\right\} d z\right)^{1 / 2}\left(\int_{|z| \geq C \sqrt{t+1}} \frac{1}{z^{2}} d z\right)^{1 / 2} \\
& =0(1) \frac{1}{(1+t)^{5 / 4}} \frac{1}{(t-t)^{1 / 4}} \leq \frac{0(1)}{|y|^{1 / 2}} \frac{1}{(1+t)^{3 / 4}} \frac{1}{(T-t)^{1 / 4}}
\end{aligned}
$$


which gives

$$
\int_{J_{1}} I(t) d t \leq 0(1) \frac{1}{|y|^{1 / 2}} .
$$

Case 2. $t \in[0, T] \cap\{t: \bar{y}>2 C \sqrt{t+1}\} \equiv J_{2}$. Then $y \geq \lambda_{j}(t+1)+2 C \sqrt{t+1}$. So

$$
\begin{aligned}
I(t)= & \left(\int_{-\infty}^{-C \sqrt{t+1}}+\int_{C \sqrt{t+1}}^{\bar{y} / 2}+\int_{\bar{y} / 2}^{+\infty}\right)\left(\frac{1}{t+1} \frac{1}{\sqrt{T-t}} \exp \left\{-\frac{(z-\bar{y})^{2}}{4(T-t)}\right\} \frac{1}{z} d z\right) \\
\leq & 0(1) \frac{1}{1+t} \frac{1}{\sqrt{T-t}} \exp \left\{-\frac{\bar{y}^{2}}{4 \mu(T-t)}\right\} \\
& \left(\int_{-\infty}^{-C \sqrt{t+1}}+\int_{C \sqrt{t+1}}^{\bar{y} / 2}\right)\left(\exp \left\{-\frac{z^{2}}{4 \mu(T-t)}\right\} \frac{1}{z}\right)+ \\
& +0(1) \frac{1}{1+t} \frac{1}{|\bar{y}|} \leq 0(1)(1+t)^{-5 / 4}(T-t)^{-\frac{1}{4}} \exp \left\{-\frac{\bar{y}^{2}}{4 \mu(T-t)}\right\}+\frac{0(1)}{1+t} \frac{1}{|\bar{y}|+1} .
\end{aligned}
$$

Consequently,

$$
\begin{aligned}
& \int_{J_{2}} I(t) d t \\
\leq & 0(1) \int_{J_{2}}(1+t)^{-5 / 4}(T-t)^{-1 / 4} \exp \left\{-\frac{\left(y-\lambda_{i}(t+1)\right)^{2}}{4 \mu(T-t)}\right\} d t \\
& +0(1) \int_{J_{2}} \frac{1}{1+t} \frac{1}{|\bar{y}|+1} d t \\
\leq & 0(1)\left[\int _ { J _ { 2 } \cap [ 0 , \frac { y } { 2 \lambda _ { i } } ] } \left((1+t)^{-5 / 4}(T-t)^{-1 / 4} \exp \left\{-\frac{\left(y-\lambda_{i}(t+1)\right)^{2}}{4 \mu(T-t)}\right\}\right.\right. \\
& \left.+\frac{1}{1+t} \frac{1}{1+|\bar{y}|}\right) d t \\
& +\int_{J_{2} \cap\left[\frac{y}{2 \lambda_{i}}, T\right]}\left((1+t)^{-5 / 4}(T-t)^{-1 / 4} \exp \left\{-\frac{\left(y-\lambda_{i}(t+1)\right)^{2}}{4 \mu(T-t)}\right\}\right. \\
& \left.\left.+\frac{1}{1+t} \frac{1}{1+|\bar{y}|}\right) d t\right] \\
\leq & 0(1)\left[T^{-\frac{1}{4}} \exp \left\{-\frac{y^{2}}{c T}\right\}+|y|^{-1} \log (1+y)+(1+|y|)^{-1 / 2}\right] .
\end{aligned}
$$

It follows from (3.67)-(3.71) that

$$
d_{p}^{-1}(y, T) I_{3}(y, T) \leq 0(1) M^{2}(T) .
$$

Finally we estimate

$$
I_{6}=0(1) M^{3}(T) \int_{0}^{t} \int H_{i}^{3}(x, t) d_{i}^{3}(x, t) \eta_{p}(x, t ; y, T) d x d t
$$


Comparing this with $I_{3}$, we see clearly that one needs only to bound the integral

$$
\begin{aligned}
& \int_{0}^{T} \int H_{p}^{3}(x, t) d_{p}^{3}(x, t) \eta_{p}(x, t ; y, T) d x d t \\
\leq & 0(1) \int_{0}^{T} \int \frac{1}{(1+t)^{3 / 2}} \frac{1}{\sqrt{T-t}} \exp \left\{-\frac{(x-y)^{2}}{4(T-t)}\right\} \frac{1}{\left(|x|^{2}+(t+1)\right)^{3 / 4}} d x d t \\
= & 0(1) \int_{0}^{T}\left(\int_{|x|<C \sqrt{t+1}}+\int_{|x| \geq C \sqrt{t+1}}\right) \\
& \left(\frac{1}{(1+t)^{3 / 2}} \frac{1}{\sqrt{T-t}} \exp \left\{-\frac{(x-y)^{2}}{4(T-t)}\right\} \frac{1}{\left(|x|^{2}+(x+1)\right)^{3 / 4}}\right) d x d t \\
\equiv & I_{61}+I_{62}
\end{aligned}
$$

These two terms can be bounded easily as follows:

$$
\begin{aligned}
I_{61} \leq & 0(1) \int_{0}^{T} \int(1+t)^{-\frac{9}{4}}(T-t)^{-1 / 2} \exp \left\{-\frac{(x-y)^{2}}{4(T-t)}\right\} \times \\
& \quad \times \exp \left\{-\frac{x^{2}}{4(t+1)}\right\} d x d t \\
\leq & 0(1) \int_{0}^{T}(1+t)^{-7 / 4}(1+T)^{-1 / 2} \exp \left\{-\frac{y^{2}}{T+1}\right\} d t \\
= & 0(1)(1+T)^{-1 / 2} \exp \left\{-\frac{y^{2}}{T+1}\right\}, \\
I_{62} \leq & \int_{0}^{T} 0(1)(1+t)^{-\frac{7}{4}}(T-t)^{-1 / 2} d t=0(1) T^{-1 / 2} .
\end{aligned}
$$

It remains to estimate $I_{62}$ for $|y| \geq C \sqrt{T+1}$. Assume $y>C \sqrt{T+1}$ (the case $y<-C \sqrt{T+1}$ is similar). Then one has

$$
\begin{aligned}
I_{62} \leq & 0(1) \int_{0}^{T}\left(\int_{-\infty}^{-C \sqrt{1+t}}+\int_{C \sqrt{1+t}}^{\frac{y}{2}}+\int_{y / 2}^{+\infty}\right) \\
& \left(\frac{1}{(1+t)^{3 / 2}} \frac{1}{\sqrt{T-t}} \exp \left\{-\frac{(x-y)^{2}}{4(T-t)}\right\} \frac{1}{\left(|x|^{2}+(t+1)\right)^{3 / 4}}\right) d x d t \\
\leq & 0(1) \int_{0}^{T}(1+t)^{-7 / 4}(T-t)^{-1 / 2} \exp \left\{-\frac{y^{2}}{4 \mu(T-t)}\right\} d t+ \\
& +0(1) \int_{0}^{T} \frac{1}{(1+t)^{3 / 2}} \int_{y / 2}^{+\infty} \frac{1}{\sqrt{T-t}} \exp \left\{-\frac{(x-y)^{2}}{4(T-t)}\right\} \frac{1}{|x|^{3 / 2}} d x \\
\leq & 0(1) T^{-1 / 2} \exp \left\{-\frac{y^{2}}{4 \mu T}\right\}+0(1)|y|^{-3 / 2}
\end{aligned}
$$

Consequently, one has

$$
d_{p}^{-1}(y, T) I_{6}(y, T) \leq 0(1) M^{3}(T) .
$$

Collecting estimates (3.61)-(3.62), (3.64)-(3.65), (3.72), and (3.74), we have shown 


\section{LEMMA 3.4. The following estimate}

$$
d_{p}^{-1}(y, T)\left|v_{p}(y, T)\right| \leq 0(1)\left\{\delta_{2}+\delta_{2}^{2}+\delta_{1}^{2}+M^{2}(T)+M^{3}(T)\right\}
$$

holds for all $y$ and $T$ such that the solution exists on $[0, T]$.

We now turn to the estimate on the derivative $\partial_{x} v_{p}(x, t)$. Using the representation formula $(3.32)_{p}$ and the proof of Lemma 3.1, one can derive by integration by parts and the symmetry in $x$ and $y$ of the scalar dual $\eta_{p}(x, t ; y, T)$ that

$$
\begin{aligned}
& \left|\partial_{y} v_{p}(y, T)\right| \\
\leq & \left|\int v_{p}(x, 0) \partial_{y} \eta_{p}(x, 0 ; y, T) d x\right|+ \\
& +\int_{0}^{T / 2} \int 0(1)|\theta(x, t)|^{2}\left|\partial_{y} \eta_{p}(x, t ; y, T)\right| d x d t+ \\
& +\int_{T / 2}^{T} \int 0(1)\left(\theta\left|\partial_{x} \theta\right|\right)(x, t) \eta_{p}(x, t ; y, T) d x d t+ \\
& +\int_{0}^{T} \int\left(|\theta| \sum_{i \neq p}\left|\partial_{x} v_{j}\right|\right)(x, t)\left|\partial_{y} \eta_{p}(x, t ; y, T)\right| d x d t+ \\
& +0(1) M^{2}(T) \int_{0}^{T} \int \sum_{i \neq p} d_{i}^{2}(x, t) H_{i}^{2}(x, t)\left|\partial_{y} \eta_{p}(x, t ; y, T)\right| d x d t+ \\
& +0(1) M^{2}(T) \int_{0}^{T} \int \sum_{i \neq p}\left(\left|\partial_{x} \rho\right|^{2} d_{i}^{2}\right)(x, t)\left|\partial_{y} \eta_{p}(x, t ; y, T)\right| d x d t \\
& +0(1) M^{3}(T) \int_{0}^{T} \int \sum_{i}\left(\left|\partial_{x} \rho\right|^{3} d_{i}^{3}\right)(x, t)\left|\partial_{y} \eta_{p}(x, t ; y, T)\right| d x d t+ \\
\equiv & +0(1) M^{3}(T) \int_{0}^{T} \int \sum_{i} H_{i}^{3}(x, t) d_{i}^{3}(x, t)\left|\partial_{y} \eta_{p}(x, t ; y, T)\right| d x d t+ \\
& +0(1)\left(\delta m_{0}+m_{0}^{2}\right) \int_{0}^{T} \int e x p\{-C(t+|x|)\}\left|\partial_{y} \eta_{p}(x, t ; y, T)\right| d x d t \\
& +0\left(\left.x\right|^{3}(x, t)\left|\partial_{y} \eta_{p}(x, t ; y, T)\right| d x d t+\right.
\end{aligned}
$$

Since many estimates on the integrals on the right hand side above can be obtained in a similar way as for $I_{i}(j \leq i \leq 7)$, so we will only sketch the estimates and point out the main differences. Here it goes. Since

$$
\begin{aligned}
I_{8}(y, T) & \leq \int\left|v_{p}(x, 0)\right|\left|\partial_{y} \eta_{p}(x, 0 ; y, T)\right| d x \\
& \leq \frac{0(1)}{\sqrt{T}} \int\left|v_{p}(x, 0)\right| \frac{1}{\sqrt{T}} \exp \left\{-\frac{(x-y)^{2}}{4 \mu T}\right\} d x
\end{aligned}
$$

so the analysis for $I_{1}$ yields

$$
d_{p}^{-1} I_{8}(y, T) \leq \frac{C}{\sqrt{T}} \delta_{2}
$$


For $y \geq C_{0}(T+1)$, (the case $y \leq-C_{0}(T+1)$ is similar), one has through integration by parts that

$$
\begin{aligned}
I_{8}= & \left|\int v_{p}(x, 0) \partial_{y} \eta_{p}(x, 0 ; y, T) d x\right| \\
= & \left|\int \partial_{x} v_{p}(x, 0) \eta_{p}(x, 0 ; y, T) d x\right| \\
\leq & \int\left|\partial_{x} v(x, 0)\right| \eta_{p}(x, 0 ; y, T) d x \\
= & \left(\int_{-\infty}^{y / 2}+\int_{y / 2}^{+\infty}\right)\left|\partial_{x} v_{p}(x, 0)\right| \eta_{p}(x, 0 ; y, T) d x \\
\leq & \frac{1}{\sqrt{4 \pi T}} \exp \left\{-\frac{y^{2}}{16 T}\right\}\left\|\partial_{x} v(\cdot, 0)\right\|_{L^{1}}+ \\
& +\left\|\partial_{x} v_{p}(\cdot, 0)\left(1+|\cdot|^{\alpha}\right)\right\|_{L^{\infty}} \int_{y / 2}^{+\infty} \frac{1}{(1+|x|)^{\alpha}} \eta_{p}(x, 0, y, T) d x \\
\leq & C \delta_{2} \frac{1}{\sqrt{4 \pi T}} \exp \left\{-\frac{y^{2}}{16 T}\right\}+C \delta_{2} \frac{1}{(1+|y|)^{\alpha}} .
\end{aligned}
$$

It follows from (3.77)-(3.78) that

$$
H_{p}^{-1}(y, T) d_{p}^{-1}(y, T) I_{8}(y, T) \leq 0(1) \delta_{2} .
$$

Next, consider

$$
\begin{aligned}
I_{9} & \equiv 0(1) \int_{0}^{\frac{T}{2}} \int|\theta(x, t)|^{2}\left|\partial_{y} \eta_{p}(x, t ; y, T)\right| d x d t \\
& \leq \frac{0(1)}{\sqrt{T}} \int_{0}^{T / 2} \int|\theta(x, t)|^{2} \frac{1}{\sqrt{T-t}} \exp \left\{-\frac{(y-x)^{2}}{4 \mu(T-T)}\right\} d x d t .
\end{aligned}
$$

So the analysis for $I_{2}$ implies that

$$
d_{p}^{-1}(y, T) I_{9}(y, T) \leq C \delta_{2}^{2} \frac{1}{\sqrt{T}}
$$

If $|y| \geq C_{0}(T+1)$, then

$$
\begin{aligned}
I_{9}(y, T) & \leq \frac{0(1) m_{0}^{2}}{\sqrt{T}} \sum_{i \neq p} \int_{0}^{\frac{T}{2}} \frac{1}{\sqrt{T(t+1)}} \exp \left\{-\frac{\left(y-\lambda_{i}(t+1)\right)^{2}}{C T}\right\} d t \\
& \leq 0(1) \delta_{2}^{2} \frac{1}{\sqrt{T}} \exp \left\{-\frac{y^{2}}{4 C T}\right\} .
\end{aligned}
$$

And so

$$
H_{p}^{-1}(y, T) d_{p}^{-1}(y, T) I_{9}(y, T) \leq 0(1) \delta_{2} .
$$

Exactly same analysis applies to

$$
I_{10} \equiv \int_{T / 2}^{T} \int 0(1)\left(|\theta|\left|\partial_{x} \theta\right|\right)(x, t) \eta_{p}(x, t) \eta_{p}(x, t ; y, T) d x d t
$$


Similarly,

$$
I_{16}(y, T) \equiv 0(1) \int_{0}^{T} \int|\theta|^{3}(x, t)\left|\partial_{y} \eta_{p}(x, t ; y, T)\right| d x d t
$$

admits the bound

$$
H_{p}^{-1}(y, T) d_{p}^{-1}(y, T) I_{16}(y, T) \leq 0(1) m_{0}^{3}=0(1) \delta_{2}^{2} .
$$

Next,

$$
\begin{aligned}
I_{11} \equiv & \int_{0}^{T} \int\left(|\theta| \sum_{j \neq p}\left|\partial_{x} v_{j}\right|\right)(x, t)\left|\partial_{y} \eta_{p}(x, t ; y, T)\right| d x d t \\
\leq & 0(1) M(T) m_{0} \int_{0}^{T} \int \sum_{i \neq p, j \neq p} d_{j}(x, t) \cdot \\
& \quad \frac{1}{\sqrt{t+1}} \exp \left\{-\frac{\left(x-\lambda_{i}(t+1)\right)^{2}}{4(t+1)}\right\} \cdot \\
& \cdot H_{j}(x, t) \frac{1}{T-t} \exp \left\{-\frac{(x-y)^{2}}{4 \mu(T-t)}\right\} d x d t \\
\leq & 0(1) M(T) m_{0} \int_{0}^{T} \sum_{i \neq p} \frac{1}{\sqrt{T}} \frac{1}{(t+1)^{3 / 4}} \frac{1}{\sqrt{T-t}} \\
& \exp \left\{-\frac{\left(y-\lambda_{i}(t+1)\right)^{2}}{4 C(T+1)}\right\} d t \\
\leq & 0(1) T^{-3 / 4}
\end{aligned}
$$

where we have used formula (3.58) and (3.51). We now assume $|y| \geq C \sqrt{T+1}$. Consider only the case $i>p$ and $y>0$ (the other cases are trivial). Then

$$
\begin{aligned}
& \int_{0}^{T} \frac{1}{\sqrt{T}} \frac{1}{(t+1)^{3 / 4}} \frac{1}{\sqrt{T-t}} \exp \left\{-\frac{\left(y-\lambda_{i}(t+1)\right)^{2}}{4 C(T+1)}\right\} d t \\
= & \left(\int_{0}^{\frac{T}{2}}+\int_{T / 2}^{T}\right) \frac{1}{\sqrt{T}} \frac{1}{(t+1)^{3 / 4}(T-t)^{1 / 2}} \exp \left\{-\frac{\left(y-\lambda_{i}(t+1)\right)^{2}}{4 C(T+1)}\right\} d t .
\end{aligned}
$$

Since

$$
\begin{aligned}
& \int_{0}^{T / 2} \frac{1}{\sqrt{T}} \frac{1}{(t+1)^{3 / 4}(T-t)^{1 / 2}} \exp \left\{-\frac{\left(y-\lambda_{i}(t+1)\right)^{2}}{4(T+1)}\right\} d t \\
\leq & \int_{0}^{\frac{y}{2 \lambda_{i}}} \frac{1}{T} \frac{1}{(t+1)^{3 / 4}} \exp \left\{-\frac{\left(y-\lambda_{i}(t+1)\right)^{2}}{4(T+1)}\right\} d t+ \\
& +\int_{\frac{y}{2 \lambda_{i}}}^{T / 2} \frac{1}{T} \frac{1}{(1+t)^{3 / 4}} \exp \left\{-\frac{\left(y-\lambda_{i}(t+1)\right)^{2}}{4(T+1)}\right\} d t \\
\leq & 0(1) T^{-3 / 4} \exp \left\{-\frac{y^{2}}{4 \mu(T+1)}\right\}+0(1) T^{-1 / 2}(1+|y|)^{-3 / 4}, \text { and } \\
& \int_{\frac{T}{2}}^{T} \frac{1}{\sqrt{T}} \frac{1}{(t+1)^{3 / 4}(T-t)^{1 / 2}} \exp \left\{-\frac{\left(y-\lambda_{i}(t+1)\right)^{2}}{4(T+1)}\right\} d t
\end{aligned}
$$




$$
\begin{aligned}
& \leq 0(1) \frac{1}{(1+T)^{3 / 4}} \int_{\frac{T}{2}}^{T} \frac{1}{\sqrt{T}} \frac{1}{\sqrt{T-t}} \exp \left\{-\frac{\left(y-\lambda_{i}(t+1)\right)^{2}}{4(T+1)}\right\} d t \\
& \leq 0(1) \frac{1}{(1+T)^{3 / 4}} \frac{1}{T^{1 / 4}},
\end{aligned}
$$

so

$$
d_{p}^{-1}(y, T) I_{11}(y, T) \leq 0(1) M(T) m_{0} T^{-1 / 2} .
$$

If $|y| \geq C_{0}(T+1)$, then it follows from (3.82) that

$$
\begin{aligned}
I_{11} & \leq 0(1) M(T) m_{0} \int_{0}^{T} \sum_{i \neq p} \frac{1}{\sqrt{T}} \frac{1}{(t+1)^{3 / 4}} \frac{1}{\sqrt{T-t}} \exp \left\{-\frac{\left(y-\lambda_{i}(t+1)\right)^{2}}{4 C(T+1)}\right\} d t \\
& =0(1) M(T) m_{0} T^{-\frac{3}{4}} \exp \left\{-\frac{\left(y-\lambda_{i}(t+1)\right)^{2}}{4 C(T+1)}\right\} .
\end{aligned}
$$

Thus

$$
H_{p}^{-1}(y, T) d_{p}^{-1}(y, T) I_{11} \leq 0(1) M(T) m_{0} \leq 0(1) \delta_{2} M(T) .
$$

As for (3.65), one can derive easily that

$$
I_{13}(y, T) \leq 0(1) M^{2}(T) T^{-1} \log (1+T) \exp \left\{-\frac{y^{2}}{\mu T}\right\}
$$

and

$$
I_{14}(y, T) \leq 0(1) M^{3}(T) T^{-1} \exp \left\{-\frac{y^{2}}{\mu T}\right\}
$$

for some positive constant $\mu>0$. Next,

$$
\begin{aligned}
I_{12}(y, T) \equiv & 0(1) M^{2}(T) \int_{0}^{T} \int \sum_{i \neq p} d_{i}^{2}(x, t) H_{i}^{2}(x, t)\left|\partial_{y} \eta_{p}(x, t ; y, T)\right| d x d t \\
= & 0(1) M^{2}(T) \sum_{i \neq p} \int_{0}^{T} \int_{\left|x-\lambda_{i}(t+1)\right| \leq C \sqrt{t+1}}\left(d_{i}^{2} H_{i}^{2}\right)(x, t)\left|\partial_{y} \eta_{p}(x, t ; y, T)\right| d x d t+ \\
& +0(1) M^{2}(T) \sum_{i \neq p} \int_{0}^{T} \int_{\left|x-\lambda_{i}(t+1)\right| \geq C \sqrt{t+1}}\left(d_{i}^{2} H_{i}^{2}\right)(x, t)\left|\partial_{y} \eta_{p}(x, t ; y, T)\right| d x d t \\
\equiv & I_{12}^{1}+I_{12}^{2} .
\end{aligned}
$$

Direct calculation using (3.58) gives

$$
\begin{aligned}
I_{12}^{1} \leq & 0(1) M^{2}(T) \sum_{i \neq p} \int_{0}^{T} \int \frac{1}{t+1} \frac{1}{\sqrt{t+1}} \exp \left\{-\frac{\left(x-\lambda_{i}(t+1)\right)^{2}}{4(t+1)}\right\} \\
& \frac{1}{T-t} \exp \left\{-\frac{(x-y)^{2}}{4 \mu(T-t)}\right\} d x d t \\
\leq & 0(1) M^{2}(T) \sum_{i \neq p} \int_{0}^{T} \frac{1}{\sqrt{T}} \frac{1}{(1+t)} \frac{1}{(T-t)^{1 / 2}} \exp \left\{-\frac{\left(y-\lambda_{i}(t+1)\right)^{2}}{4 C T}\right\} d t
\end{aligned}
$$


which can be estimated as for $I_{11}$. To estimate $I_{12}^{2}$, we first note that by following the analysis for $I_{32}$ (see (3.68)-(3.71)), one can check easily that

$$
d_{p}^{-1}(y, T) I_{12}^{2}(y, T) \leq 0(1) M^{2}(T) T^{-1 / 2} .
$$

So it remains to study the case that $|y| \geq C_{0}(T+1)$. We will only consider the integral

$$
\int_{0}^{T} \int_{\left|x-\lambda_{i}(t+1)\right| \geq C \sqrt{t+1}}\left(d_{i}^{2} H_{i}^{2}\right)(x, t) \mid \partial_{y} \eta_{p}(x, t ; y, T) d x d t
$$

for $i>p$ and $y<-C_{0}(T+1)$. The other cases can be treated similarly. Setting $\bar{y}=y-\lambda_{i}(t+1)$, one can bound the above integral by

$$
\begin{aligned}
& 0(1) \int_{0}^{T} \int_{|z| \geq C \sqrt{t+1}} K^{2}(z, t) \frac{1}{|z|} \frac{1}{T-t} \exp \left\{-\frac{(z-\bar{y})^{2}}{4 \mu(T-t)}\right\} d z d t \\
= & 0(1) \int_{0}^{T}\left(\int_{-\infty}^{\bar{y} / 2}+\int_{\bar{y} / 2}^{-C \sqrt{t+1}}+\int_{C \sqrt{t+1}}^{+\infty}\right)\left(K^{2}(z, t)\right) \frac{1}{|z|} \frac{1}{T-t} \\
& \exp \left\{-\frac{(z-\bar{y})^{2}}{4 \mu(T-t)}\right\} d z d t .
\end{aligned}
$$

The last two integrals are bounded by

$$
\begin{aligned}
& 0(1) \int_{0}^{T} \frac{\exp \left\{-\frac{\bar{y}^{2}}{C(T-t)}\right\}}{(t+1) \sqrt{T-t}}\left(\int_{\bar{y} / 2}^{-C \sqrt{t+1}}+\int_{C \sqrt{t+1}}^{+\infty}\right)\left(\frac{1}{|z|} \frac{1}{\sqrt{T-t}}\right) \times \\
& \times \exp \left\{-\frac{(\bar{y}-z)^{2}}{C(T-t)}\right\} d z d t \\
& =0(1) \int_{0}^{T} \frac{1}{(1+t)^{5 / 4}} \frac{1}{(T-t)^{3 / 4}} \exp \left\{-\frac{\bar{y}^{2}}{C(T-t)}\right\} d t \\
& =0(1) T^{-3 / 4} \exp \left\{-\frac{y^{2}}{4 \mu T}\right\} \text {, }
\end{aligned}
$$

while the first integral is estimated by

$$
\begin{aligned}
& 0(1) \int_{0}^{T} K^{2}\left(\frac{\bar{y}}{2}, t\right) \frac{1}{|\bar{y}|} \frac{1}{\sqrt{T-t}} d t \\
= & 0(1)\left(\int_{0}^{|y| / C_{1}}+\int_{|y| / C_{1}}^{T}\right) K^{2}\left(\frac{\bar{y}}{2}, t\right) \frac{1}{|\bar{y}|} \frac{1}{\sqrt{T-t}} d t \\
\leq & 0(1) \int_{0}^{|y| / C_{1}} \frac{1}{|\bar{y}|+t+1} \frac{1}{|\bar{y}|} \frac{1}{\sqrt{T-t}} d t+ \\
& +\int_{|y| / C_{1}}^{T} \frac{1}{t+1} \frac{1}{|\bar{y}|} \frac{1}{\sqrt{T-t}} d t \\
= & 0(1)|y|^{-3 / 2},
\end{aligned}
$$

where we have chosen $C_{1}$ so that $t \leq|y| / C_{1}$ implies $\left|\frac{\bar{y}}{2}\right| \geq C_{0}(t+1)$. Thus we have shown that for $|y| \geq C_{0}(T+1)$

$$
I_{12}^{2}(y, T) \leq 0(1) M^{2}(T)|y|^{-3 / 2} .
$$


It follows that

$$
H_{p}^{-1}(y, T) d_{p}^{-1}(y, T) I_{12}(y, T) \leq 0(1) M^{2}(T)
$$

Comparing

$$
I_{15} \equiv 0(1) M^{3}(T) \int_{0}^{T} \int \sum_{i} H_{i}^{3}(x, t) d_{i}^{3}(x, t)\left|\partial_{y} \eta_{p}(x, t ; y, T)\right| d x d t
$$

with $I_{12}$ and modifying the estimate for $I_{6}$ slightly, one easily shows that

$$
H_{p}^{-1}(y, T) d_{p}^{-1}(y, T) I_{15}(y, T) \leq 0(1) M^{3}(T)
$$

Since $I_{17}$ is estimated trivially as for $I_{7}$, we have shown that

LEMMA 3.5. For all $y$ and $T>0$,

$$
d_{p}^{-1}(y, T) H_{p}^{-1}(y, T)\left|\partial_{y} v_{p}(y, T)\right| \leq 0(1)\left(\delta_{2}+\delta_{2}^{2}+\delta_{1}^{2}+M^{2}(T)+M^{3}(T)\right)
$$

This completes the analysis for wave in the principal field.

3.4. A priori estimate II - on transversal waves. In this section, we treat the waves moving in the transversal directions. The estimates are somewhat more complicated than the ones for the principal waves due to the errors induced by the approximate scalar dual waves. We start with the basic estimate on $v_{i}(x, t)$.

The representation formula $(3.31)_{i}$ for $i \neq p$ gives

$$
\begin{aligned}
\left|v_{i}(y, T)\right| \leq & \int\left|v_{i}(x, 0)\right| \eta_{i}(x, 0 ; y, T) d x+ \\
& +0(1) M(T) \int_{0}^{T} \int\left|E_{i}(x, t ; y, T)\right| d_{i}(x, t) d x d t+ \\
& +\int_{0}^{T} \int 0(1) \sum_{j \neq i}\left|\theta_{j}\right|^{2}(x, t) \eta_{i}(x, t ; y, T) d x d t+ \\
& +0(1) M(T) \int_{0}^{T} \int \sum_{j \neq p}\left(\left|\partial_{x} \rho\right| d_{j}\right)(x, t) \eta_{i}(x, t ; y, T) d x d t+ \\
& +0(1) M(T) \int_{0}^{T} \int\left(\sum_{j \neq p} d_{j} H_{j}\left|\partial_{x} \rho\right|\right)(x, t) \eta_{i}(x, t ; y, T) d x d t+ \\
& +0(1) M(T) \int_{0}^{T} \int \sum_{j}\left(|\theta| H_{j} d_{j}\right)(x, t) \eta_{i}(x, t ; y, T) d x d t+ \\
& +0(1) M^{2}(T) \int_{0}^{T} \int \sum_{j \neq p}\left(H_{j}^{2} d_{j}^{2}\right)(x, t) \eta_{i}(x, t ; y, T) d x d t+ \\
& +0(1) M^{2}(T) \int_{0}^{T} \int \sum_{j \neq p}\left(d_{j} d_{p} H_{p} H_{j}\right)(x, t) \eta_{i}(x, t ; y, T) d x d t+ \\
& +0(1) M^{2}(T) \int_{0}^{T} \int \sum_{j \neq p}\left(d_{j} d_{p} H_{p}\left|\partial_{x} \rho\right|\right)(x, t) \eta_{i}(x, t ; y, T) d x d t+
\end{aligned}
$$




$$
\begin{aligned}
& +0(1) M^{2}(T) \int_{0}^{T} \int \sum_{j \neq p}\left(\left|\partial_{x} \rho\right|^{2} d_{j}^{2}\right)(x, t) \eta_{i}(x, t ; y, T) d x d t+ \\
& +0(1) \int_{0}^{T} \int\left(\left|\partial_{x} w(x, t)\right|^{3}+|\theta(x, t)|^{3}\right) \eta_{i}(x, t ; y, T) d x d t+ \\
& +0(1)\left(\delta m_{0}+m_{0}^{2}\right) \int_{0}^{T} \int \exp \{-C(t+|x|)\} \eta_{i}(x, t ; y, T) d x d t \\
& \equiv \sum_{i=1}^{12} K_{i}
\end{aligned}
$$

with $K_{i}(1 \leq i \leq 11)$ denote in order the integrals on the right hand side of (3.91). Each integral $K_{i}$ will be estimated below. We will make full use of Lemmas 3.2-3.3, in particular, the estimate

$$
\eta_{i}(x, t ; y, T) \leq \frac{1}{\sqrt{4 \pi(T-t)}} \exp \left\{-\frac{\eta_{1}(x-\bar{x}(t, y, T))^{2}}{4(T-t)}\right\}
$$

which follows from (3.42) in Lemma 3.3. We will always assume $i>p$ (the case $i<p$ is similar). First,

$$
K_{1} \leq\|\eta(\cdot, 0 ; y, T)\|_{L^{\infty}}\left\|v_{i}(\cdot, 0)\right\|_{L^{1}} \leq 0(1) \delta_{2} \frac{1}{\sqrt{T}} .
$$

If $|y| \geq C_{0}(T+1)$, then it follows from Lemma 3.3 that

$$
|\bar{x}(0, y, T)| \geq \frac{1}{2}|y|+0(1) \sqrt{T+1}
$$

and so

$$
\begin{aligned}
K_{1} \leq & \int_{-\infty}^{\bar{x} / 2} \eta_{i}(x, 0 ; y, T)\left|v_{i}(x, 0)\right| d x+ \\
& +\int_{\frac{\bar{x}}{2}}^{+\infty} \eta_{i}(x, 0 ; y, T)\left|v_{i}(x, 0)\right|\left(1+|x|^{1 / 2}\right) \frac{d x}{1+|x|^{1 / 2}} \\
\leq & 0(1) \delta_{2} \frac{1}{(1+|y|)^{1 / 2}}
\end{aligned}
$$

Thus

$$
d_{i}^{-1}(y, T) K_{1}(y, T) \leq 0(1) \delta_{2} .
$$

Next we turn to the estimate of the integral involving the errors due to that $\eta_{i}$ does not satisfy the scalar dual equation (3.23) exactly. Using (3.33)-(3.34) of Lemma 3.2, we have

$$
\begin{aligned}
& K_{2}(y, T) \\
\equiv & 0(1) M(T) \int_{0}^{T} \int\left|E_{i}(x, t ; y, T)\right| d_{i}(x, t) d x d t \\
\leq & 0(1) M(T)\left[\int_{0}^{T} \int\left(\left|\partial_{x} \rho\right| d_{i}\right)(x, t) \widetilde{\eta}_{i}(x, t ; y, T) d x d t+\right.
\end{aligned}
$$




$$
\begin{aligned}
& +\int_{0}^{T} \int\left(\left|\partial_{x} \rho\right| d_{i}\right)(x, t) \tilde{\eta}_{i}(x, t ; y, T)(T-t)^{-\frac{1}{2}} d x d t+ \\
& \left.+\int_{0}^{T} \int \delta(T-t)^{-\frac{1}{2}}(1+t)^{-1 / 2} \widetilde{\eta}_{i}(x, t ; y, T) d_{i}(x, t) d x d t\right] \equiv K_{2}^{1}+K_{2}^{2}+K_{2}^{3}
\end{aligned}
$$

To treat $K_{2}^{1}$, one notes that

$$
\begin{aligned}
& \exp \left\{-\frac{x^{2}}{4 \mu^{\prime}(t+1)}\right\} \exp \left\{-\frac{(x-\bar{x})^{2}}{4 \mu(T-t)}\right\} \\
= & \exp \left\{-\frac{\bar{x}^{2}}{4\left[\mu(T-t)+\mu^{\prime}(t+1)\right]}\right\} \\
& \exp \left\{-\frac{\mu(T-t)+\mu^{\prime}(t+1)}{4 \mu \mu^{\prime}(t+1)(T-t)}\left(x-\frac{\mu^{\prime}(t+1) \bar{x}}{\mu(T-t)+\mu^{\prime}(t+1)}\right)^{2}\right\},
\end{aligned}
$$

and

$$
\exp \left\{-\frac{x^{2}}{8(t+1)}\right\} d_{i}(x, t) \leq 0(1)(1+t)^{1 / 2} \quad(i \neq p)
$$

Thus

$$
\begin{aligned}
K_{2}^{1} \leq & 0(1) M(T) \delta \int_{0}^{T} \int \exp \left\{-\frac{x^{2}}{8(t+1)}\right\} d_{i}(x, t) \frac{1}{\sqrt{(t+1)(T-t)}} \\
& \exp \left\{-\frac{x^{2}}{8(t+1)}\right\} \exp \left\{-\frac{(x-\bar{x})^{2}}{4 \mu(T-t)}\right\} d x d t \\
\leq & 0(1) M(T) \delta \int_{0}^{T} \frac{1}{\sqrt{T}} \frac{1}{\sqrt{t+1}} \exp \left\{-\frac{\bar{x}^{2}}{4 C T}\right\} d t .
\end{aligned}
$$

If $\left|y-\lambda_{i}(T+1)\right| \leq C \sqrt{T+1}$, then $\bar{x}=y-\lambda_{i}(T-t) \bmod (0(1) \sqrt{T+1})$ by Lemma 3.3. And so

$$
\begin{aligned}
K_{2}^{1} & \leq 0(1) M(T) \delta \int_{0}^{T} \frac{1}{\sqrt{T}} \frac{1}{\sqrt{t+1}} \exp \left\{-\frac{\left(y-\lambda_{i}(T-t)\right)^{2}}{4 C T}\right\} d t \\
& \leq 0(1) M(T) \delta \int_{0}^{T} \frac{1}{\sqrt{T}} \frac{1}{\sqrt{t+1}} \exp \left\{-\frac{(t+1)^{2}}{4 C^{\prime} T}\right\} d t=0(1) T^{-\frac{1}{4}}
\end{aligned}
$$

Assume now that $y>\lambda_{i}(T+1)+C \sqrt{T+1}$. Thus $\bar{x}=y-\lambda_{i}(T-t)+0(1) \sqrt{T+1}$. Recall that $i>p$. We thus have

$$
\begin{aligned}
K_{2}^{1} & \leq 0(1) M(T) \delta \int_{0}^{T} \frac{1}{\sqrt{T}} \frac{1}{\sqrt{t+1}} \exp \left\{-\frac{\left(y-\lambda_{i}(T+1)+\lambda_{i}(t+1)\right)^{2}}{4 C T}\right\} \\
& \leq 0(1) M(T) \delta T^{-\frac{1}{4}} \exp \left\{-\frac{\left(y-\lambda_{i}(T+1)\right)^{2}}{4 C T}\right\} .
\end{aligned}
$$

If $0 \leq y \leq \lambda_{i}(T+1)-C \sqrt{T+1}$, it then follows from (3.45)-(3.46) of Lemma 3.3 that 


$$
\begin{aligned}
K_{2}^{1} & \leq 0(1) M(T) \delta \int_{0}^{T} \frac{1}{\sqrt{T}} \frac{1}{\sqrt{t+1}} \exp \left\{-\frac{\bar{x}^{2}}{4 C T}\right\} d t \\
& \leq 0(1) M(T) \delta \int_{0}^{T} \frac{1}{\sqrt{T}} \frac{1}{\sqrt{t+1}} \exp \left\{-\frac{(t-\bar{t})^{2}}{4 C^{\prime} T}\right\} d t \\
& =0(1) M(T) \delta\left(\int_{0}^{\bar{t} / 2}+\int_{\bar{t} / 2}^{T}\right)\left(\frac{1}{\sqrt{T}} \frac{1}{\sqrt{t+1}} \exp \left\{-\frac{(t-\bar{t})^{2}}{4 C^{\prime} T}\right\}\right) d t \\
& \leq 0(1) M(T) \delta T^{-1 / 4} \exp \left\{-\frac{\left(y-\lambda_{i}(T+1)\right)^{2}}{4 \mu T}\right\}+ \\
& \quad+0(1) M(T) \delta \frac{1}{\left(1+\left|y-\lambda_{i}(T+1)\right|\right)^{1 / 2}} .
\end{aligned}
$$

Finally for $y<0$, one has by (3.41) of Lemma 3.3 that

$$
|\bar{x}(t, y, T)|=|y|+\left|\lambda_{i}\left(x_{1}, t\right)\right|(T-t) .
$$

So direct calculation leads to

$$
\begin{aligned}
K_{2}^{1} & \leq 0(1) M(T) \delta \int_{0}^{T} \frac{1}{\sqrt{T}} \frac{1}{\sqrt{t+1}} \exp \left\{-\frac{\bar{x}^{2}}{4 C T}\right\} d t \\
& \leq 0(1) \frac{1}{\sqrt{T}} \exp \left\{-\frac{y^{2}}{4 \mu T}\right\} .
\end{aligned}
$$

In summary we have shown

$$
d_{i}^{-1}(y, T) K_{2}^{1}(y, T) \leq 0(1) \delta M(T) .
$$

Similar (even easier) analysis shows

$$
d_{i}^{-1}(y, T) K_{2}^{2}(y, T) \leq 0(1) \delta M(T) .
$$

It remains to bound $K_{2}^{3}$. First we have

$$
\begin{aligned}
K_{2}^{3} & \equiv 0(1) M(T) \delta \int_{0}^{T} \int(T-t)^{-1 / 2}(1+t)^{-1 / 2} \tilde{\eta}_{i}(x, t ; y, T) d_{i}(x, t) d x d t \\
& \leq 0(1) M(T) \delta \int_{0}^{T}(T-t)^{-1 / 2}(1+t)^{-\frac{3}{4}}\left\|\widetilde{\eta}_{i}(\cdot, t ; y, T)\right\|_{L^{1}} d t \\
& \leq 0(1) M(T) \delta T^{-1 / 4} .
\end{aligned}
$$

Note that

$$
\begin{aligned}
K_{2}^{3} \leq & 0(1) M(T) \delta \int_{0}^{T} \int(T-t)^{-1 / 2}(1+t)^{-3 / 4} \tilde{\eta}_{i}(x, t ; y, T) \\
& \exp \left\{-\frac{\left(x-\lambda_{i}(t+1)^{2}\right.}{4(t+1)}\right\} d x d t+ \\
& +0(1) M(T) \delta \int_{0}^{T} \int_{\left|x-\lambda_{i}(t+1)\right| \geq C \sqrt{t+1}}(T-t)^{-1 / 2}(1+t)^{-1 / 2} \\
& \widetilde{\eta}_{i}(x, t ; y, T)\left|x-\lambda_{i}(t+1)\right|^{-\frac{1}{2}} d x \\
\equiv & 0(1) M(T) \delta\left(J_{3}+J_{4}\right) .
\end{aligned}
$$


Directly calculation gives

$$
J_{3} \leq 0(1) \int_{0}^{T} \frac{1}{\sqrt{T}}(T-t)^{-1 / 2}(1+t)^{-\frac{1}{4}} \exp \left\{-\frac{\left(\bar{x}-\lambda_{i}(t+1)\right)^{2}}{4 C T}\right\} d t .
$$

If $y \geq \lambda_{i}(T+1)+C \sqrt{T+1}$, then $\bar{x}-\lambda_{i}(t+1)=y-\lambda_{i}(T+1)+0(1) \sqrt{T+1}$ by Lemma 3.3. So

$$
J_{3} \leq 0(1) T^{-1 / 4} \exp \left\{-\frac{\left(y-\lambda_{i}(T+1)\right)^{2}}{4 \mu T}\right\} .
$$

If $0 \leq y<\lambda_{i}(T+1)-C \sqrt{T+1}$, then $0<\bar{t} \leq T$, and by Lemma 3.3 one has

$$
\begin{aligned}
J_{3} \leq & 0(1)\left(\int_{0}^{\bar{t}}+\int_{\bar{t}}^{T}\right)\left(T^{-1 / 2}(T-t)^{-1 / 2}(1+t)^{-\frac{1}{4}} \exp \left\{-\frac{\left(\bar{x}-\lambda_{i}(t+1)\right)^{2}}{4 C T}\right\} d t\right. \\
\leq & 0(1) \int_{0}^{\bar{t}} T^{-1 / 2}(T-t)^{-1 / 2}(1+t)^{-\frac{1}{4}} \exp \left\{-\frac{\bar{x}^{2}}{4 C T}\right\} \exp \left\{-\frac{(t+1)^{2}}{4 \mu T}\right\} d t \\
& +0(1) \int_{\bar{t}}^{T} T^{-1 / 2}(T-t)^{-1 / 2}(1+t)^{-\frac{1}{4}} \exp \left\{-\frac{\left(\bar{x}-\lambda_{i}(t+1)\right)^{2}}{4 C T}\right\} d t .
\end{aligned}
$$

The second integral is estimated as in the previous step since $\bar{x} \geq 0$. While the first integral above is bounded in the same ways as for $K_{2}^{1}$. Since the case for $y<0$ is trivial, we obtain the desired estimate for $J_{3}$. We now turn to the estimate on $J_{4}$.

$$
J_{4}=\int_{0}^{T} \frac{1}{\sqrt{t+1}} \frac{1}{T-t} d t \int_{|z| \geq C \sqrt{t+1}} \exp \left\{-\frac{(z-\widetilde{x})^{2}}{4 \mu(T-t)}\right\}|z|^{-1 / 2} d z
$$

with $\widetilde{x}=\bar{x}(t, y, T)-\lambda_{i}(t+1)$. If $y \geq \lambda_{i}(T+1)+4 C \sqrt{T+1}$, then $\widetilde{x}=y-\lambda_{i}(T+$ 1) $+0(1) \delta \sqrt{T+1} \geq 2 C \sqrt{T+1}$ for small $\delta$ by Lemma 3.3. Thus

$$
\begin{aligned}
J_{4}= & \int_{0}^{T} \frac{1}{\sqrt{t+1}} \frac{1}{T-t}\left(\int_{-\infty}^{-C \sqrt{t+1}}+\int_{C \sqrt{t+1}}^{\frac{\check{x}}{2}}+\int_{\frac{\tilde{x}}{2}}^{+\infty}\right) \\
& \exp \left\{-\frac{(z-\tilde{x})^{2}}{4 \mu(T-t)}\right\} \frac{1}{|z|^{1 / 2}} d t \\
\leq & \int_{0}^{T} \frac{1}{(1+t)^{3 / 4}} \frac{1}{(T-t)^{1 / 2}} \exp \left\{-\frac{\widetilde{x}^{2}}{4 C(T-t)}\right\} d t+ \\
& +\int_{0}^{T} \frac{1}{\sqrt{t+1}} \frac{1}{\sqrt{T-t}} \frac{1}{\sqrt{\widetilde{x}}} d x \\
\leq & \frac{0(1)}{\left|y-\lambda_{i}(T+1)\right|^{1 / 2}}+0(1) T^{-1 / 4} \exp \left\{-\frac{\left(y-\lambda_{i}(T+1)\right)^{2}}{4 \mu T}\right\} .
\end{aligned}
$$

Now assume $z \leq \lambda_{i}(T+1)-4 C \sqrt{T+1}$. We will consider only the case that $0<y \leq$ $\lambda_{i}(T+1)-4 C \sqrt{T+1}$, since $y<0$ is treated similarly. Rewrite $J_{4}$ as

$$
\begin{aligned}
J_{4}= & \left(\int_{0}^{t_{1}}+\int_{t_{1}}^{\bar{t}}+\int_{\bar{t}}^{T}\right)\left(\frac{1}{\sqrt{t+1}} \frac{1}{T-t}\right) . \\
& \cdot \int_{|z| \geq C \sqrt{t+1}} \exp \left\{-\frac{(z-\widetilde{x})^{2}}{4 \mu(T-t)}\right\}|z|^{-1 / 2} d z d t,
\end{aligned}
$$


where $t_{1}$ is a fixed constant such that $\lambda_{i}(t+1) \geq 2 C \sqrt{t+1}$ for $t \geq t_{1}$. The first integral in (3.100) is clearly bounded by $0(1) T^{-1 / 2}$. The third integral is estimated in a similar way as in (3.99) by using the fact $\widetilde{x}=y-\lambda_{i}(T+1)+0(1) \delta \sqrt{T+1} \leq$ $-2 C \sqrt{T+1}$ for $t \geq \bar{t}$ as follows from Lemma 3.3, and dividing the space integral as $\int_{-\infty}^{\frac{\tilde{x}}{2}}+\int_{\frac{\tilde{x}}{2}}^{-C \sqrt{t+1}}+\int_{C \sqrt{t+1}}^{+\infty}$. Now for $t \in\left[t_{1}, \bar{t}\right], \bar{x} \leq 0$, so $|\widetilde{x}|=|\bar{x}|+\lambda_{i}(t+1) \geq$ $\sqrt{\mu_{3}}(\bar{t}-t)+\lambda_{i}(t+1) \geq \mu_{4} \bar{t}$ by Lemma 3.3. Consequently,

$$
\begin{aligned}
& \int_{t_{1}}^{\bar{t}} \frac{1}{\sqrt{t+1}} \frac{1}{T-t} \int_{|z| \geq C \sqrt{t+1}} \exp \left\{-\frac{(z-\bar{x})^{2}}{4 \mu(T-t)}\right\}|z|^{-1 / 2} d z d t \\
= & \int_{t_{1}}^{\bar{t}} \frac{1}{\sqrt{t+1}} \frac{1}{T-t}\left(\int_{-\infty}^{\frac{\tilde{x}}{2}}+\int_{\frac{\tilde{x}}{2}}^{-C \sqrt{t+1}}+\int_{C \sqrt{t+1}}^{+\infty}\right) \exp \left\{-\frac{(z-\bar{x})^{2}}{4 \mu(T-t)}\right\}|z|^{-1 / 2} d z d t \\
\leq & 0(1) \int_{t_{1}}^{\bar{t}} \frac{1}{\sqrt{t+1}} \frac{1}{\sqrt{T-t}} \frac{1}{|\widetilde{t}|^{1 / 2}} d t+ \\
& +0(1) \int_{t_{1}}^{\bar{t}} \frac{1}{\sqrt{t+1}} \frac{1}{\sqrt{T-t}} \frac{1}{(1+t)^{1 / 4}} \exp \left\{-\frac{\widetilde{x}^{2}}{4 C(T-t)}\right\} d t \\
\leq & 0(1)\left|y-\lambda_{i}(T+1)\right|^{-1 / 2}+0(1) T^{-\frac{1}{4}} \exp \left\{-\frac{\left|y-\lambda_{i}(T+1)\right|^{2}}{4 C T}\right\} .
\end{aligned}
$$

We conclude from the above estimates that for $\left|y-\lambda_{i}(T+1)\right| \geq 4 C \sqrt{T+1}$,

$$
J_{3}+J_{4} \leq 0(1)\left|y-\lambda_{i}(T+1)\right|^{-1 / 2} .
$$

It follows from (3.97)-(3.98) and (3.101) that

$$
d_{i}^{-1}(y, T) K_{2}^{3}(y, T) \leq 0(1) \delta M(T) .
$$

This combined with (3.95)-(3.96) yields the desired estimates

$$
d_{i}^{-1}(y, T) K_{2}(y, T) \leq 0(1) \delta M(T)
$$

Next, we bound

$$
K_{3}(y, T)=0(1) \sum_{j \neq i} \int_{0}^{T} \int\left|\theta_{j}\right|^{2}(x, t) \eta_{i}(x, t ; y, T) d x d t .
$$

Direct computation leads to that for $j<i,(j>i$ is similar $)$,

$$
\begin{aligned}
& \int_{0}^{T} \int\left|\theta_{j}\right|^{2}(x, t) \eta_{i}(x, t ; y, T) d x d t \\
\leq & 0(1) m_{0}^{2} \int_{0}^{T} \int \frac{1}{(t+1) \sqrt{T-t}} \exp \left\{-\frac{\left(x-\lambda_{j}(t+1)\right)^{2}}{2(t+1)}\right\} \times \\
& \times \exp \left\{-\frac{(x-\bar{x})^{2}}{4 \mu(T-t)}\right\} d x d t \\
\leq & 0(1) m_{0}^{2} \int_{0}^{T} \frac{1}{\sqrt{T} \sqrt{t+1}} \exp \left\{-\frac{\left(\bar{x}-\lambda_{j}(t+1)\right)^{2}}{4 C(T+1)}\right\} d t
\end{aligned}
$$


which is clearly bounded by $0(1) m_{0}^{2} T^{-\frac{1}{4}}$ if $\left|y-\lambda_{i}(T+1)\right| \leq C \sqrt{T+1}$ and by $0(1) m_{0}^{2} T^{-1} \exp \left\{-\frac{\left(y-\lambda_{i}(T+1)\right)^{2}}{4 C(T+1)}\right\}$ if $y \geq \lambda_{i}(T+1)+C \sqrt{T+1}$ since in this case $\bar{x}-$ $\lambda_{j}(t+1)=y-\lambda_{i}(T+1)+\left(\lambda_{i}-\lambda_{j}\right)(t+1)+0(1) \sqrt{T+1}$. Now assume $0 \leq y \leq$ $\lambda_{i}(T+1)-C \sqrt{T+1}$ (the case $y<0$ is trivial). Then

$$
\begin{aligned}
& \int_{0}^{T} \frac{1}{\sqrt{T} \sqrt{t+1}} \exp \left\{-\frac{\left(\bar{x}-\lambda_{j}(t+1)\right)^{2}}{4 C(T+1)}\right\} d t \\
= & \left(\int_{0}^{\bar{t}}+\int_{\bar{t}}^{T}\right) \frac{1}{\sqrt{T} \sqrt{t+1}} \exp \left\{-\frac{\left(\bar{x}-\lambda_{j}(t+1)\right)^{2}}{4 C(T+1)}\right\} d t .
\end{aligned}
$$

Clearly

$$
\begin{aligned}
& \int_{\bar{t}}^{T} \frac{1}{\sqrt{T}} \frac{1}{\sqrt{t+1}} \exp \left\{-\frac{\left(\bar{x}-\lambda_{j}(t+1)\right)^{2}}{4 C(T+1)}\right\} d t \\
\leq & 0(1) \frac{1}{\sqrt{1+\bar{t}}} \int_{\bar{t}}^{T} \frac{1}{\sqrt{T}} \exp \left\{-\frac{\left(\left(\lambda_{i}-\lambda_{j}\right)(t+1)+\left(y-\lambda_{i}(T+1)\right)\right)^{2}}{4 C(T+1)}\right\} \\
\leq & 0(1) \frac{1}{\sqrt{1+\left|y-\lambda_{i}(T+1)\right|}}
\end{aligned}
$$

while

$$
\int_{0}^{\bar{t}} \frac{1}{\sqrt{T}} \frac{1}{\sqrt{t+1}} \exp \left\{-\frac{\left(\bar{x}-\lambda_{j}(t+1)\right)^{2}}{4 C(T+1)}\right\} d t \leq \frac{0(1)}{\sqrt{1+\left|y-\lambda_{i}(T+1)\right|}}
$$

where we have used $\bar{x} \leq 0$ for $t \leq \bar{t},(3.46)$, and $\frac{\partial}{\partial t}\left(\bar{x}-\lambda_{j}(t+1)\right)=\lambda_{i}(\bar{x}, t)-\lambda_{j}+0(1) \delta \neq$ 0 for small $\delta$. It follows that

$$
d_{i}^{-1}(y, T) K_{3}(y, T) \leq 0(1) \delta_{2}^{2}
$$

We now consider

$$
K_{4}(y, T) \equiv 0(1) M(T) \int_{0}^{T} \int \sum_{j \neq p}\left(\left|\partial_{x} \rho\right| d_{j}\right)(x, t) \eta_{i}(x, t ; y, T) d x d t .
$$

It should be clear that one needs only to treat the integral

$$
\int_{0}^{T} \int\left|\partial_{x} \rho\right|(x, t) d_{j}(x, t) \eta_{i}(x, t ; y, T) d x d t
$$

for $j \neq p$. But this is carried out exactly as for $K_{2}^{1}$ to obtain

$$
d_{i}^{-1}(y, T) K_{4}(y, T) \leq 0(1) M(T) \delta_{1} .
$$

Similarly, one has

$$
d_{i}^{-1}(y, T) K_{5}(y, T) \leq 0(1) M(T) \delta_{1}
$$

where

$$
K_{5}(y, T) \equiv 0(1) M(T) \int_{0}^{T} \int\left(\sum_{j \neq p} d_{j} H_{j}\left|\partial_{x} \rho\right|\right)(x, t) \eta_{i}(x, t ; y, T) d x d t .
$$


Next, consider

$$
K_{6}(y, T)=0(1) M(T) \int_{0}^{T} \int \sum_{j}\left(|\theta| H_{j} d_{j}\right)(x, t) \eta_{i}(x, t ; y, T) d x d t .
$$

The only nontrivial integral in $K_{6}$ is

$$
\begin{aligned}
& \int_{0}^{T} \int\left(\left|\theta_{i}\right| H_{i} d_{i}\right)(x, t) \eta_{i}(x, t ; y, T) d x d t \\
\leq & 0(1) m_{0} \int_{0}^{T} \int \frac{1}{(t+1) \sqrt{T-t}} \exp \left\{-\frac{\left(x-\lambda_{i}(t+1)\right)^{2}}{4(t+1)}\right\} \times \\
& \times \exp \left\{-\frac{(x-\bar{x})^{2}}{4 \mu(T-t)}\right\} d_{i}(x, t) d x d t \\
\leq & 0(1) m_{0} \int_{0}^{T} \frac{1}{(1+t)^{3 / 4}} \frac{1}{\sqrt{T}} \exp \left\{-\frac{\left(\bar{x}-\lambda_{i}(t+1)\right)^{2}}{4 C(T+1)}\right\} d t \\
\leq & 0(1) m_{0} T^{-1 / 4} \exp \left\{-\frac{\left|y-\lambda_{i}(T+1)\right|^{2}}{4 \mu(T+1)}\right\} \text { for all } y \geq 0,
\end{aligned}
$$

by following the analysis for $K_{3}(y, T)$. It follows that

$$
d_{i}^{-1}(y, T) K_{6}(y, T) \leq 0(1) M(T) m_{0} \leq 0(1) M(T) \delta_{2} .
$$

We next turn to the integral

$$
\int_{0}^{T} \int \sum\left(H_{j}^{2} d_{j}^{2}\right)(x, t) \eta_{i}(x, t ; y, T) d x d t
$$

For fixed $j$,

$$
\begin{aligned}
& \int_{0}^{T} \int\left(H_{j}^{2} d_{j}^{2}\right)(x, t) \eta_{i}(x, t ; y, T) d x d t \\
= & \int_{0}^{T}\left(\int_{\left|x-\lambda_{j}(t+1)\right| \leq C \sqrt{t+1}}+\int_{\left|x-\lambda_{j}(t+1)\right| \geq C \sqrt{t+1}}\right)\left(H_{j}^{2} d_{j}^{2}\right)(x, t) \eta_{i}(x, t ; y, T) d x d t \\
\equiv & J_{5}+J_{6} .
\end{aligned}
$$

Now,

$$
J_{5} \leq 0(1) \int_{0}^{T} \int \frac{1}{(t+1)^{3 / 2} \sqrt{T-t}} \exp \left\{-\frac{\left(x-\lambda_{j}(t+1)\right)^{2}}{4(t+1)}\right\} \exp \left\{-\frac{(x-\bar{x})^{2}}{4(T-t)}\right\} d x d t
$$

and similar (even easier) analysis as for $K_{3}$ and $K_{6}$ applies. It thus remains to estimate $J_{6}$. First, one has

$$
\begin{aligned}
J_{6} & =\int_{0}^{T} \int_{\left|x-\lambda_{j}(t+1)\right| \geq C \sqrt{t+1}}\left(H_{j}^{2} d_{j}^{2}\right)(x, t) \eta_{i}(x, t ; y, T) d x d t \\
& \leq 0(1) \int_{0}^{T}(1+t)^{-5 / 4}(T-t)^{-\frac{1}{4}} d t=0(1) T^{-1 / 4} .
\end{aligned}
$$


So we will consider only the case that $\left|y-\lambda_{i}(T+1)\right| \geq 4 C \sqrt{T+1}$. Rewrite $J_{6}$ as

$$
J_{6} \leq 0(1) \int_{0}^{T} \frac{1}{t+1} \frac{1}{\sqrt{T-t}} \int_{|z| \geq C \sqrt{t+1}} \exp \left\{-\frac{(z-\tilde{x})^{2}}{4 \mu(T-t)}\right\} \frac{d z}{|z|} d t
$$

with $\widetilde{x}=\bar{x}(t ; y, T)-\lambda_{j}(t+1)$. If $y>\lambda_{i}(T+1)+4 C \sqrt{T+1}$, then $\widetilde{x}=\left(y-\lambda_{i}(T+\right.$ 1)) $+\left(\lambda_{i}-\lambda_{j}\right)(t+1)+0(1) \delta \sqrt{T+1}$. The easy case that $j \leq i$ is treated as in (3.99). For $j>i$,

$$
\begin{aligned}
J_{6} \leq 0(1) & \left(\int_{\{\widetilde{x} \leq 2 C \sqrt{t+1}\}}+\int_{\{\widetilde{x} \geq 2 C \sqrt{t+1}\}}\right) \frac{d t}{(t+1) \sqrt{T-t}} \times \\
& \times \int_{|z| \geq C \sqrt{t+1}} \exp \left\{-\frac{(z-\bar{x})^{2}}{4 \mu(T-t)}\right\} \frac{d z}{|z|}
\end{aligned}
$$

Clearly,

$$
\begin{aligned}
& \int_{\{\tilde{x} \leq 2 C \sqrt{t+1}\}} \frac{d t}{(t+1) \sqrt{T-t}} \int_{|z| \geq C \sqrt{t+1}} \exp \left\{-\frac{(z-\tilde{x})^{2}}{4 \mu(T-t)}\right\} \frac{d z}{|z|} \\
\leq & 0(1) \int_{\{\tilde{x} \leq 2 C \sqrt{t+1}\}} \frac{d t}{(t+1)^{5 / 4}(T-t)^{\frac{1}{4}}} \\
\leq & \frac{0(1)}{\left|y-\lambda_{i}(T+1)\right|^{1 / 2}} \int \frac{d t}{(1+t)^{3 / 4}(T-t)^{1 / 4}},
\end{aligned}
$$

while

$$
\begin{aligned}
& \int_{\{\tilde{x}>2 C \sqrt{t+1}\}} \frac{d t}{(t+1) \sqrt{T-t}} \int_{|z| \geq C \sqrt{t+1}} \exp \left\{-\frac{(z-\tilde{x})^{2}}{4 \mu(T-t)}\right\} \frac{d z}{|z|} \\
= & \int_{\{\tilde{x}>2 C \sqrt{t+1}\}} \frac{d t}{(t+1) \sqrt{T-t}}\left(\int_{-\infty}^{-C \sqrt{t+1}}+\int_{C \sqrt{t+1}}^{\frac{\tilde{x}}{2}}+\int_{\frac{\tilde{x}}{2}}^{+\infty}\right) \\
& \exp \left\{-\frac{(z-\tilde{x})^{2}}{4 \mu(T-t)}\right\} \frac{d z}{|z|} \\
\leq & \int_{\{\tilde{x}>2 C \sqrt{t+1}\}} \frac{0(1)}{(1+t)^{5 / 4}(T-t)^{1 / 4}} \exp \left\{-\frac{\tilde{x}^{2}}{4 C T}\right\} d t+ \\
& +\int_{\{\tilde{x}>2 C \sqrt{t+1}\}} \frac{0(1)}{1+t} \frac{1}{|\widetilde{x}|} d t \\
\leq & 0(1)\left|y-\lambda_{i}(T+1)\right|^{-1 / 2} .
\end{aligned}
$$

We next treat the case that $0 \leq y \leq \lambda_{i}(T+1)-4 C \sqrt{T+1}$. By Lemma 3.3 it is easily verified that there exists a fixed constant $\sigma>0$ so that

$$
\widetilde{x}<0,|\widetilde{x}| \geq 2 C \sqrt{t+1} \text { for all } t \in[0, \sigma \bar{t}]
$$


provided that $\delta$ is small. Thus

$$
\text { (3.115) } \begin{aligned}
J_{6} \leq & 0(1)\left(\int_{0}^{\sigma \bar{t}}+\int_{\sigma \bar{t}}^{T}\right) \frac{1}{\sqrt{T-1}} \frac{1}{t+1} \int_{|z| \geq C \sqrt{t+1}} \exp \left\{-\frac{(z-\tilde{x})^{2}}{4 \mu(T-t)}\right\} \frac{d z}{|z|} d t \\
\leq & 0(1) \int_{\sigma \bar{t}}^{T} \frac{1}{(t+1)^{5 / 4}} \frac{d t}{(T-t)^{\frac{1}{4}}}+0(1) \int_{0}^{\sigma \bar{t}} \frac{d t}{(t+1) \sqrt{T-t}} \\
& \left(\int_{-\infty}^{\frac{x}{2}}+\int_{\frac{\tilde{x}}{2}}^{-C \sqrt{t+1}}+\int_{C \sqrt{t+1}}^{+\infty}\right) \exp \left\{-\frac{(z-\tilde{x})^{2}}{4 \mu(T-t)}\right\} \frac{d z}{|z|} \\
\leq & 0(1)\left|y-\lambda_{i}(T+1)\right|^{-1 / 2} .
\end{aligned}
$$

We conclude from above estimates on $J_{5}$ and $J_{6}$ that

$$
d_{i}^{-1}(y, T) \int_{0}^{T} \int \sum_{j}\left(H_{j}^{2} d_{j}^{2}\right)(x, t) \eta_{i}(x, t ; y, T) d x d t \leq 0(1) .
$$

Consequently,

$$
d_{i}^{-1}(y, T)\left[K_{7}+K_{8}\right] \leq 0(1) M^{2}(T) .
$$

The rest of $K$ 's can be treated easily. For example,

$$
\begin{aligned}
K_{9} \equiv & 0(1) M^{2}(T) \int_{0}^{T} \int\left(\sum_{j} d_{j} d_{p}\left|\partial_{x} \rho\right|\right)(x, t) \eta_{i}(x, t ; y, T) d x d t \\
\leq & 0(1) M^{2}(T) \int_{0}^{T} \int\left(\sum_{j} d_{j}^{2}\left|\partial_{x} \rho\right|^{2}\right) \eta_{i}(x, t ; y, T) d x d t+ \\
& +0(1) M^{2}(T) \int_{0}^{T} \int\left(d_{p}^{2} H_{p}^{2}\right)(x, t) \eta_{i}(x, t ; y, T) d x d t \\
\leq & 0(1) M^{2}(T) d_{i}(y, T)+0(1) M^{2} \delta^{2} d_{i}(y, T)
\end{aligned}
$$

as follows from (3.116) and the similar analysis for $K_{4}$;

$$
\begin{aligned}
K_{10} & \equiv 0(1) M^{2}(T) \int_{0}^{T} \int \sum_{j \neq p}\left(\left|\partial_{x} \rho\right|^{2} d_{j}^{2}\right)(x, t) \eta_{i}(x, t ; y, T) d x d t \\
& \leq 0(1) M^{2}(T) \delta^{2} d_{i}(y, T)
\end{aligned}
$$

by the estimate for $K_{4}$;

$$
\begin{aligned}
K_{11} \equiv & 0(1) \int_{0}^{T} \int\left(\left|\partial_{x} w(x, t)\right|^{3}+|\theta(x, t)|^{3}\right) \eta_{i}(x, t ; y, T) d x d t \\
\leq & 0(1) M^{3}(T)\left[\int_{0}^{T} \int \sum\left(d_{j}^{3} H_{j}^{3}\right)(x, t) \eta_{i}(x, t ; y, T) d x d t\right. \\
& \left.+\int_{0}^{T} \int\left(\sum\left|\partial_{x} \rho\right|^{3} d_{j}^{3}\right)(x, t) \eta_{i}(x, t ; y, T) d x d t\right]+ \\
& +0(1) \int_{0}^{T} \int \sum_{j} \theta_{j}^{3}(x, t) \eta_{i}(x, t ; y, T) d x d t \\
\leq & 0(1)\left(M^{3}(T)+\delta^{3}+m_{0}^{3}\right) d_{i}(y, T)
\end{aligned}
$$


and finally,

$$
\begin{aligned}
K_{12} & \equiv 0(1)\left(\delta m_{0}+m_{0}^{2}\right) \int_{0}^{T} \int \exp \{-C(t+|x|)\} \eta_{i}(x, t ; y, T) d x d t \\
& \leq 0(1)\left(\delta m_{0}+m_{0}^{2}\right) d_{i}(y, T)
\end{aligned}
$$

through direct computation.

Collecting all the estimates, (3.93), (3.103), (3.107)-(3.110), and (3.117)-(3.121), we arrive at

LEMMA 3.6. For $\delta$ suitably small, one has that

$$
d_{i}^{-1}(y, T)\left|v_{i}(y, T)\right| \leq 0(1)\left(\delta_{2}+\delta_{1}^{2}+\delta_{2}^{2}+M^{2}(T)+M^{3}(T)\right)
$$

for all $(y, T) \in \mathbb{R}^{1} \times(0, \infty)$ and $i \neq p$.

This completes the basic estimate for the transversal waves. Next we proceed to derive the necessary a priori estimates on the derivatives of the transversal waves in order to complete the bound on $M(t)$ defined in (3.52). It follows from representation formula $(3.32)_{i}$ and Lemma 3.1 that

$$
\begin{aligned}
\left|\partial_{i} v_{i}(y, T)\right| \leq & \left|\int v_{0}(x, 0) \partial_{y} \eta_{i}(x, 0 ; y, T) d x\right|+ \\
& +\left|\int_{0}^{T} \int_{\partial_{y}} E_{i}(t, x ; y, T) v_{i}(x, t) d x d t\right| \\
& +0(1) \int_{0}^{T / 2} \int \sum_{j \neq i} \theta_{j}^{2}(x, t)\left|\partial_{y} \eta_{i}(x, t ; y, T)\right| d x d t+ \\
& +0(1) \int_{\frac{T}{2}}^{T} \int\left(\sum_{j \neq i}\left|\theta_{j}\right| \partial_{x} \theta_{j} \mid\right)(x, t) \eta_{i}(x, t ; y, T) d x d t+ \\
& +0(1) \int_{0}^{T / 2} \int \sum_{j \neq p}\left(\left|v_{j}\right|\left|\partial_{x} \rho\right|\right)(x, t)\left|\partial_{y} \eta_{i}(x, t ; y, T)\right| d x d t+ \\
& +0(1) \int_{\frac{T}{2}}^{T} \int \sum_{j \neq p}\left(\left|v_{j}\right|\left(\left|\partial_{x} \rho\right|^{2}+\left|\partial_{x}^{2} \rho\right|\right)\right)(x, t) \widetilde{\eta}(x, t ; y, T) d x d t+ \\
& +0(1) \int_{0}^{T} \int \sum_{j \neq p}\left(\left|\partial_{x} v_{j}\right|\left|\partial_{x} \rho\right|\right)(x, t)\left|\partial_{y} \eta_{i}(x, t ; y, T)\right| d x d t+ \\
& +0(1)\left(\delta m_{0}+m_{0}^{2}\right) \int_{0}^{T} \int \exp \{-(1+|x|)\}\left|\partial_{y} \eta_{i}(x, t ; y, T)\right| d x d t+ \\
& +0(1) \int_{0}^{T} \int\left(|\theta|\left|\partial_{x} v\right|\right)(x, t)\left|\partial_{y} \eta_{i}(x, t ; y, T)\right| d x d t+ \\
& +0(1) \int_{0}^{T} \int\left|\partial_{x} v\right|^{2}(x, t)\left|\partial_{y} \eta_{i}(x, t ; y, T)\right| d x d t+ \\
& +0(1) \int_{0}^{T} \int \sum_{j \neq p}\left(\left|\partial_{x} \rho\right|^{2} v_{j}^{2}\right)(x, t)\left|\partial_{y} \eta_{i}(x, t ; y, T)\right| d x d t+ \\
& \left.+\left|\partial_{x} w(x, t)\right|^{3}+|\theta(x, t)|^{3}\right)\left|\partial_{y} \eta_{i}(x, t ; y, T)\right| d x d t+ \\
& \\
& \\
& \\
&
\end{aligned}
$$




$$
\begin{aligned}
& +0(1)\left(\delta m_{0}+m_{0}^{2}\right) \int_{T / 2}^{T} \int \exp \{-C(t+|x|)\} \tilde{\eta}_{i}(x, t ; y, T) d x d t \\
\equiv & \sum_{j=13}^{25} K_{j}
\end{aligned}
$$

where $\tilde{\eta}_{i}$ is bounded as in (3.34), and we have used (3.39) and integrations by parts in the derivation of (3.123). Each $K_{j},(13 \leq j \leq 25)$, denoting in order the integrals on the right hand side of (3.123), will be estimated separately. We will always assume $i>p$. Note that

$$
\begin{aligned}
\left|\partial_{y} \eta_{i}(x, t ; y, T)\right| & \leq 0(1) \frac{1}{\sqrt{T-t}} \tilde{\eta}_{i}(x, t ; y, T) \\
& \equiv \frac{0(1)}{\sqrt{T-t}} \frac{1}{\sqrt{4 \pi(T-t)}} \exp \left\{-\frac{m_{i}^{2}(x, t ; y, T)}{4 \mu(T-t)}\right\}
\end{aligned}
$$

with some fixed $\mu>0$. First, the analysis for $K_{1}$, gives

$$
\left|K_{13}(y, T)\right|=\left|\int v_{0}(x, 0) \partial_{y} \eta_{i}(x, 0 ; y, T) d x\right| \leq 0(1) T^{-\frac{1}{2}} d_{i}(y, T) .
$$

For $\left|y-\lambda_{i}(T+1)\right| \geq C_{0}(T+1)$, we can obtain by using (3.39) and integration by parts that

$$
\begin{aligned}
\left|K_{13}(y, T)\right| \leq & \left|\int \partial_{x} v_{i}(x, 0) \frac{\lambda_{i}(x, 0)}{\lambda_{i}(y, 0)} \eta_{i}(x, 0 ; y, T) d x\right|+ \\
& +0(1) \int\left|v_{i}(x, 0)\right| \widetilde{\eta}_{i}(x, 0, y, T)\left|\partial_{x} \rho(x, 0)\right| d x \\
\leq & 0(1) \delta_{2}\left(1+\left|y-\lambda_{i}(T+1)\right|\right)^{-\alpha}
\end{aligned}
$$

by the same argument as in (3.78). It follows from (3.125)-(3.126) that

$$
H_{i}^{-1}(y, T) d_{i}^{-1}(y, T) K_{13}(y, T) \leq 0(1) \delta_{2} .
$$

Next, we bound $K_{14}$ by

$$
\begin{aligned}
K_{14} & \equiv\left|\int_{0}^{T} \int \partial_{y} E_{i}(t, x ; y, T) v_{i}(x, t) d x d t\right| \\
& \leq\left|\int_{0}^{T / 2} \int \partial_{y} E_{i}(t, x ; y, T) v_{i}(x, t) d x d t\right|+\left|\int_{\frac{T}{2}}^{T} \int \partial_{y} E_{i}(x, t ; y, T) v_{i}(x, t) d x d t\right|,
\end{aligned}
$$

which we denote in order by $K_{14}^{1}$ and $K_{14}^{2}$ respectively. Note first that

$$
\partial_{y} E_{i}(x, t ; y, T)=\frac{1}{\sqrt{T-t}} \bar{E}_{i}(x, t ; y, T)
$$

with $\bar{E}_{i}(x, t ; y, T)$ satisfying bound (3.33) as for $E_{i}$, which follows direct computation using (3.30). Thus

$$
\begin{aligned}
K_{14}^{1}(y, T) & \leq \int_{0}^{\frac{T}{2}} \int\left|\partial_{y} E_{i}(t, x ; y, T)\right|\left|v_{i}(x, t)\right| d x d t \\
& \leq \int_{0}^{\frac{T}{2}} \frac{1}{\sqrt{T-t}} \int\left|\bar{E}_{i}(x, t ; y, T)\right| \\
& \leq 0(1) \frac{1}{\sqrt{T}} M(T) \delta_{1} d_{i}(y, T)
\end{aligned}
$$


as follows from the analysis on $K_{2}$. As for $K_{14}^{2}$, we use (3.35)-(3.36) in Lemma 3.2 to obtain

$$
\begin{aligned}
K_{14}^{2}(y, T)= & \left|\int_{\frac{T}{2}}^{T} \int \partial_{y} E_{i}(x, t ; y, T) v_{i}(x, t) d x d t\right| \\
\leq & \int_{\frac{T}{2}}^{T} \int \frac{\left|\lambda_{i}(x, t)\right|}{\left|\lambda_{i}(y, t)\right|}\left|\partial_{x} v_{i}(x, t)\right|\left|E_{i}(x, t ; y, T)\right| d x d t+ \\
& +\int_{\frac{T}{2}}^{T} \int \frac{\left|\partial_{x} \lambda_{i}(x, t)\right|}{\left|\lambda_{i}(y, t)\right|}\left|v_{i}(x, t)\right|\left|E_{i}(x, t ; y, T)\right| d x d t+ \\
& +\int_{\frac{T}{2}}^{T} \int\left|v_{i}(x, t)\right|\left|\widetilde{E}_{i}(x, t ; y, T)\right| d x d t \\
\leq & 0(1) M(T) T^{-1 / 2} \int_{\frac{T}{2}}^{T} \int\left(H_{i}(x, t)+\left|\partial_{x} \rho(x, t)\right|\right)(1+t)^{1 / 2} \\
& d_{i}(x, t)\left|E_{i}(x, t ; y, T)\right| d x d t+ \\
& +0(1) M(T) T^{-1 / 2} \int_{\frac{T}{2}}^{T} \int\left|d_{i}(x, t)\right|\left|(t+1)^{1 / 2} \widetilde{E}_{i}(x, t ; y, T)\right| d x d t \\
\leq & 0(1) M(T) T^{-1 / 2} d_{i}(y, T) \delta_{1}
\end{aligned}
$$

Thus,

$$
T^{\frac{1}{2}} d_{i}^{-1}(y, T) K_{14}(y, T) \leq 0(1) \delta_{1} M(T)
$$

To obtain the faster decay of $K_{14}(y, T)$ for $\left|y-\lambda_{i}(T+1)\right| \geq C_{0}(T+1)$, we rewrite $K_{14}$ as

$$
\begin{aligned}
K_{14}(y, T) \leq & 0(1) M(T) \int_{0}^{T} \int\left(H_{i} d_{i}\right)(x, t)\left|E_{i}(x, t ; y, T)\right| d x d t+ \\
& +0(1) M(T) \int_{0}^{T} \int\left|\partial_{x} \rho\right| d_{i}(x, t)\left|E_{i}(x, t ; y, T)\right| d x d t \\
& +0(1) M(T) \int_{0}^{T} \int d_{i}(x, t)\left|\tilde{E}_{i}(x, t ; y, T)\right| d x d t
\end{aligned}
$$

By the structures of $E_{i}$ and $\widetilde{E}_{i}$ and the estimate of $K_{2}$ for $\left|y-\lambda_{i}(T+1)\right| \geq C_{0}(T+1)$, we see clearly that the least non-trivial term on the right hand side of (3.132) is

$$
0(1) M(T) \delta \int_{0}^{T} \int H_{i}(x, t) d_{i}(x, t)(T-t)^{-1 / 2}(1+t)^{-1 / 2} \widetilde{\eta}_{i}(x, t ; y, T) d x d t .
$$

By inspecting the estimate for $K_{3}$, one needs only to bound the integral

$$
J_{7}=\int_{0}^{T} \int \frac{1}{\sqrt{t+1}} \frac{1}{T-t} \int_{|z| \geq C \sqrt{t+1}} \exp \left\{-\frac{(z-\tilde{x})^{2}}{4 \mu(T-t)}\right\} K(z, t)|z|^{-1 / 2} d z d t
$$

with $\widetilde{x}=\bar{x}(t, y, T)-\lambda_{i}(t+1)$. We will only consider that $i>p$ and $C_{0}(T+1) \leq y \leq$ $C_{1}(T+1)$ for any fixed constant $C_{1}>C_{0}$, since the other cases can be treated similarly. Then in this case, $\bar{x}=y-\lambda_{i}(T+1)+0(1) \delta \sqrt{T+1}$. Set $\beta=3-2 \alpha$. Then $\frac{1}{2}<\beta<1$ 
since $1<\alpha<5 / 4$. Fix a positive constant $C_{2}$ so that $C_{2}(1+T)^{\beta}+0(1) \delta \sqrt{1+T} \geq$ $\frac{1}{2} C_{2}(1+T)^{\beta}$. Rewrite $J_{7}$ as

$$
\begin{aligned}
J_{7}= & \left(\int_{0}^{T-C_{2}(1+T)^{\beta}}+\int_{T-C_{2}(1+T)^{\beta}}\right) \\
& \frac{d t}{\sqrt{t+1}(T-t)} \int_{|z| \geq C \sqrt{t+1}} \exp \left\{-\frac{(z-\widetilde{x})^{2}}{4 \mu(T-t)}\right\} K(z, t)|z|^{-1 / 2} d z \\
\equiv & J_{71}+J_{72}
\end{aligned}
$$

For $t \in\left[0, T-C_{2}(1+T)^{\beta}\right], \widetilde{x} \geq C_{0}(t+1)+C_{1}(1+T)^{\beta}+0(1) \delta(1+T)^{1 / 2} \geq C_{0}(t+1)+$ $\frac{C_{1}}{2}(1+T)^{\beta}$. And so $K\left(\widetilde{x}-\frac{C_{2}}{2}(1+T)^{\beta}, t\right)=\left(\left|\widetilde{x}-\frac{C_{2}}{2}(1+t)^{\beta}\right|^{2 \alpha-1}+(t+1)\right)^{-1 / 2}$ by definition of $K$ (see (3.51)). Consequently,

$$
\begin{aligned}
J_{71} \equiv & \int_{0}^{T-C_{2}(1+T)^{\beta}} \frac{d t}{\sqrt{t+1}(T-t)}\left[\left(\int_{-\infty}^{-C \sqrt{t+1}}+\int_{+C \sqrt{t+1}}^{\widetilde{x}-\frac{C_{2}}{2}(1+T)^{\beta}}\right)\right. \\
& \left.+\int_{\widetilde{x}-\frac{C_{2}}{2}(1+T)^{\beta}}^{+\infty}\right] \exp \left\{-\frac{(z-\widetilde{x})^{2}}{4 \mu(T-t)}\right\} K(z, t)|z|^{-1 / 2} d z \\
\leq & 0(1) \int_{0}^{T-C_{2}(1+T)^{\beta}} \frac{1}{(1+t)^{5 / 4} \sqrt{T-t}} \exp \left\{-\frac{C_{2}^{2}(1+T)^{2 \beta}}{32(T-t)}\right\} d t \\
& +0(1) \int_{0}^{T-C_{2}(1+T)^{\beta}} \frac{1}{\sqrt{t+1} \sqrt{T-t}}\left|\widetilde{x}-\frac{C_{2}}{2}(1+T)^{\beta}\right|^{-\alpha} d t \\
\leq & 0(1)\left|y-\lambda_{i}(T+1)\right|^{-\alpha}
\end{aligned}
$$

Since $\exp \left\{-\frac{C_{2}(1+T)^{2 \beta}}{32(T-t) \mu}\right\} \leq \exp \left\{-\frac{C_{2}^{2}(1+T)^{2 \beta-1}}{32 \mu}\right\}$ and $2 \beta>1$. Next,

$$
\begin{aligned}
J_{72} \equiv & \int_{T-C_{2}(1+T)^{\beta}}^{T} \frac{d t}{(T-t) \sqrt{t+1}}\left[\left(\int_{-\infty}^{-C \sqrt{t+1}}+\int_{C \sqrt{t+1}}^{\widetilde{x}-\frac{C_{2}}{2}(1+T)^{\beta}}\right)\right. \\
& \left.+\int_{\widetilde{x}-\frac{C_{2}}{2}(1+T)^{\beta}}\right] \exp \left\{-\frac{(z-\widetilde{x})^{2}}{4 \mu(T-t)}\right\} K(z, t)|z|^{-1 / 2} d z \\
\leq & \int_{T-C_{2}(1+T)^{\beta}}^{T} \frac{0(1)}{(1+t)^{5 / 4} \sqrt{T-t}} \exp \left\{-\frac{C_{2}(1+T)^{\beta}}{32 \mu}\right\} d t \\
& \left.+\int_{T-C_{2}(1+T)^{\beta}}^{T} \frac{0(1)}{(1+t) \sqrt{T-t}} \frac{d \tilde{x}-\left.\frac{C_{2}}{2}(1+T)^{\beta}\right|^{-1 / 2}}{32 \mu}\right\} \\
= & 0(1)(1+T)^{-\frac{5}{4}+\frac{\beta}{2}} \exp \left\{-\frac{C_{2}(1+T)^{\beta}}{32 \mu}\right. \\
& +0(1)(1+T)^{-1+\frac{\beta}{2}}\left|\widetilde{x}-\frac{C_{2}}{2}(1+T)^{\beta}\right|^{-1 / 2} \\
= & 0(1)\left|y-\lambda_{i}(T+1)\right|^{-\alpha}
\end{aligned}
$$

It follows that

$$
H_{i}^{-1} d_{i}^{-1}(y, T) K_{14}(y, T) \leq 0(1) \delta_{1} M(T)
$$


Next, since

$$
\begin{aligned}
K_{15} & =0(1) \int_{0}^{\frac{T}{2}} \int \sum_{j \neq i} \theta_{j}^{2}(x, t)\left|\partial_{y} \eta_{i}(x, t ; y, T)\right| d x d t \\
& =0(1) T^{-1 / 2} \int_{0}^{\frac{T}{2}} \int \sum_{j \neq i} \theta_{j}^{2} \tilde{\eta}_{i} d x d t, \\
K_{16} & =0(1) \int_{T / 2}^{T} \int\left(\sum_{j \neq i}\left|\theta_{j}\right|\left(\partial_{x} \theta_{j}\right)\right)(x, t) \eta_{i}(x, t ; y, T) d x d t \\
& =0(1) T^{-1 / 2} \int_{T / 2}^{T} \int \sum_{j \neq i} \theta_{j}^{2} \eta_{i} d x d t,
\end{aligned}
$$

so (3.124) and the analysis for $K_{3}$ leads to

$$
H_{i}^{-1} d_{i}^{-1}(y, T)\left(K_{15}(y, T)+K_{16}(y, T)\right) \leq 0(1) \delta_{2}^{2} .
$$

Similarly

$$
\begin{aligned}
K_{17}(y, T) & \equiv 0(1) \int_{0}^{T / 2} \int \sum_{j \neq p}\left(\left|v_{j}\right|\left|\partial_{x} \rho\right|\right)(x, t)\left|\partial_{y} \eta_{i}(x, t ; y, T)\right| d x d t \\
& \leq 0(1) M(T) T^{-1 / 2} \int_{0}^{\frac{T}{2}} \int \sum_{j \neq p}\left(\left|\partial_{x} \rho\right| d_{j}\right)(x, t)\left|\widetilde{\eta}_{i}(x, t ; y, T)\right| d x d t
\end{aligned}
$$

so the analysis for $K_{2}^{1}$ yields

$$
H_{i}^{-1} d_{i}^{-1} K_{7}(y, T) \leq 0(1) M(T) \delta_{1},
$$

and

$$
\begin{aligned}
(3.139) K_{18}(y, T) & \equiv 0(1) \int_{T / 2}^{T} \int \sum_{j \neq p}\left(\left|v_{j}\right|\left(\left|\partial_{x} \rho\right|^{2}+\left|\partial_{x}^{2} \rho\right|\right)(x, t) \tilde{\eta}_{i}(x, t ; y, T) d x d t\right. \\
& \leq 0(1) M(T) T^{-1 / 2} \int_{T / 2}^{T} \int \sum_{j \neq p}\left(\left|\partial_{x} \rho\right| d_{i}\right)(x, t) \tilde{\eta}_{i}(x, t ; y, T) d x d t \\
& \leq 0(1) \delta M(T)^{-1 / 2} d_{i}(y, T) H_{i}(y, T) .
\end{aligned}
$$

Consider now

$$
\begin{aligned}
K_{19}(y, T) & \equiv 0(1) \int_{0}^{T} \int \sum_{j \neq p}\left(\left|\partial_{x} \rho\right|\left|\partial_{x} v_{j}\right|\right)(x, t)\left|\partial_{y} \eta_{i}(x, t ; y, T)\right| d x d t \\
& \leq 0(1) M(T) \int_{0}^{T} \frac{1}{\sqrt{(T-t)(t+1)}} \sum_{j \neq p} \int d_{j}(x, t)\left|\partial_{x} \rho(x, t)\right| \eta_{i}(x, t ; y, T) d x d t
\end{aligned}
$$

So by a trivial modification of the estimate for $K_{2}^{1}$ shows that

$$
H_{i}^{-1}(y, T) d_{i}^{-1}(y, T) K_{19}(y, T) \leq 0(1) \delta M(T) .
$$


Following the analysis for $K_{2}^{1}, K_{3}$ and $K_{6}$, we see that

$$
\begin{aligned}
K_{20}(y, T) \equiv & 0(1) \int_{0}^{T} \int\left(|\theta|\left|\partial_{x} v\right|\right)(x, t)\left|\partial_{y} \eta_{i}(x, t ; y, T)\right| d x d t \\
= & 0(1) M(T) \int_{0}^{T} \frac{1}{\sqrt{(T-t)(t+1)}} \int \\
& \sum_{j}\left(|\theta| d_{j}\right)(x, t)\left|\tilde{\eta}_{i}(x, t ; y, T)\right| d x d t \\
\leq & 0(1) M(T) m_{0} H_{i}(y, T) d_{i}(y, T) .
\end{aligned}
$$

We now turn to estimate the integrals involving the nonlinear terms. First,

$$
\begin{aligned}
K_{21}(y, T) & \equiv 0(1) \int_{0}^{T} \int\left|\partial_{x} v(x, t)\right|^{2}\left|\partial_{y} \eta_{i}(x, t ; y, T)\right| d x d t \\
& \leq 0(1) M^{2}(T) \int_{0}^{T} \frac{d t}{\sqrt{T-t}} \int \sum_{j}\left(H_{j}^{2} d_{j}^{2}\right)(x, t) \tilde{\eta}_{i}(x, t ; y, T) d x d t
\end{aligned}
$$

Now one can repeat the derivation (3.116) almost line by line with obvious modifications to show that

$$
K_{21}(y, T) \leq 0(1) M^{2}(T) d_{i}(y, T) H_{i}(y, T) .
$$

Next, as in the estimates for $K_{17}, K_{18}$, and $K_{19}$, we can obtain

$$
\begin{aligned}
K_{22}(y, T) \equiv & 0(1) \int_{0}^{T} \int\left(\sum_{j \neq p}\left|\partial_{x} \rho\right|^{2}\left|v_{j}\right|^{2}\right)(x, t)\left|\partial_{y} \eta_{i}(x, t ; y, T)\right| d x d t \\
\leq & 0(1) M^{2}(T) \delta_{1} \int_{0}^{T} \frac{1}{\sqrt{(t+t)(T-1)}} \sum_{j \neq p} \\
& \int d_{j}^{2}(x, t)\left|\partial_{x} \rho(x, t)\right|\left|\tilde{\eta}_{i}(x, t ; y, T)\right| d x d t \\
\leq & 0(1) M^{2}(T) \delta_{1}^{2} d_{i}(y, T) H_{i}(y, T) .
\end{aligned}
$$

The higher order terms can be handled easily. Indeed,

$$
\begin{aligned}
K_{23}(y, T) \equiv & 0(1) \int_{0}^{T} \int\left(\left|\partial_{x} w(x, t)\right|^{2}+|\theta(x, t)|^{3}\right)\left|\partial_{y} \eta_{i}(x, t ; y, T)\right| d x d t \\
\leq & 0(1) M^{3}(T)\left[\int_{0}^{T} \frac{d t}{(1+t)^{3 / 2}(T-t)^{1 / 2}} \int \sum_{j} d_{j}^{3}(x, t) \widetilde{\eta}_{i}(x, t ; y, T) d x d t+\right. \\
& \left.+\int_{0}^{T} \frac{d t}{(T-t)^{1 / 2}} \int \sum_{j} d_{j}^{3}(x, t)\left|\partial_{x} \rho(x, t)\right|^{3} \widetilde{\eta}_{i}(x, t ; y, T) d x\right]+ \\
& +0(1) \int_{0}^{T} \frac{d t}{(T-t)^{1 / 2}} \int \sum_{j \neq p} \theta_{j}^{3}(x, t) \widetilde{\eta}_{i}(x, t ; y, T) d x .
\end{aligned}
$$

The first two integrals on the right hand side above can be estimated as in (3.142) for $K_{21}(y, T)$, while the last integral above is estimated as for $K_{20}$ (see (3.141)). Thus

$$
K_{23}(y, T) \leq\left(0(1) M^{3}(T)\left(1+\delta_{1}^{3}\right)+0(1) m_{0}^{3}\right) d_{i}(y, T) H_{i}(y, T) .
$$


Finally, the integrals involving exponentially decay terms can be estimated trivially to obtain

$$
\begin{aligned}
K_{24}(y, T)+K_{25}(y, T) \equiv & 0(1)\left(\delta m_{0}+m_{0}^{2}\right) \\
& {\left[\int _ { 0 } ^ { T } \int \operatorname { e x p } \left\{-C(|t|+|x|)\left|\partial_{y} \eta_{i}(x, t ; y, T)\right| d x d t\right.\right.} \\
& \left.+\int_{T / 2}^{T} \int \exp \{-C(t+|x|)\} \widetilde{\eta}_{i}(x, t ; y, T) d x d t\right] \\
\leq & 0(1)\left(\delta_{1}^{2}+m_{0}^{2}\right) d_{i}(y, T) H_{i}(y, T) .
\end{aligned}
$$

Collecting all the estimates we have obtained thus far, (3.125), (3.131), (3.136)(3.145), we conclude that

LEMMA 3.7. For all $y \in \mathbb{R}^{1}, T>0$, it holds that

$$
\left|\partial_{y} v_{i}(y, T)\right| \leq 0(1)\left(\delta_{2}+\delta_{2}^{2}+\delta_{2}^{2}+M^{2}(T)+M^{3}(T)\right) d_{i}(y, T) H_{i}(y, T), \quad i \neq p
$$
provided that $\delta_{1}$ is suitably small.

This completes the a priori estimate on the transversal waves.

3.5. Proof of the stability theorem. With the a priori estimates derived in the previous two subsections, we can now complete the proof of our main theorem easily. Indeed, it follows from Lemmas 3.4-3.7 and (3.51)-(3.53) that

$$
M(T) \leq 0(1)\left(\delta_{2}+\delta_{2}^{2}+\delta_{1}^{2}+M^{2}(T)+M^{3}(T)\right)
$$

provided that $\delta=\left|u_{+}-u_{-}\right| \leq \delta_{1}$ is suitably small. So if $M(T), \delta_{1}$ and $\delta_{2}$ are small, we then have

$$
M(T) \leq 0(1)\left(\delta_{1}+\delta_{2}\right)
$$

Furthermore, we note that (3.8) implies

$$
M(0) \leq 0(1)\left(\delta_{1}+\delta_{2}\right)
$$

Consequently,

$$
M(t) \leq 0(1)\left(\delta_{1}+\delta_{2}\right) \text { for } t \geq 0
$$

and for suitably small $\delta_{1}$ and $\delta_{2}$ by continuity. We thus have shown

Proposition 3.8. (A priori estimate.) Let T be any positive constant. Suppose $w(x, t)$ is a smooth solution to the Cauchy problem (3.4)-(3.8) defined on the time interval $[0, T]$ and $v(x, t)$ is the corresponding characteristic variable (3.9). Let $M(t)$ be defined by (3.52). Then there exist positive constant $\delta_{0}$ and $C$ independent of time $T$ so that if $\delta_{1}+\delta_{2} \leq \delta_{0}$, then

$$
M(t) \leq C\left(\delta_{1}+\delta_{2}\right) \quad \text { for all } t \in[0, T],
$$

and

$$
\begin{aligned}
\left|v_{i}(x, t)\right| \leq & C\left(\delta_{1}+\delta_{2}\right)\left(\left|x-\lambda_{i}(t+1)\right|^{2}+(t+1)\right)^{-\frac{1}{4}} \\
\left|\partial_{x} v_{i}(x, t)\right| \leq & C\left(\delta_{1}+\delta_{2}\right)\left(\chi\left(\left(x-\lambda_{i}\right)(t+1)\right), t\right)\left|x-\lambda_{i}(t+1)\right|^{2 \alpha-1} \\
& +(t+1)^{-\frac{1}{2}}\left(\left|x-\lambda_{i}(t+1)\right|^{2}+(t+1)\right)^{-\frac{1}{4}}
\end{aligned}
$$

for all $t \in[0, T]$, where $\chi(x, t)$ is the characteristic function of the interval $\left[-C_{0}(t+1), C_{0}(t+1)\right]$.

Now the theorem follows from Proposition 3.8 by the standard argument for parabolic equations [9]. Thus the proof of the theorem is complete. 
4. An application. In this section we will show that our general theory can be applied to an interesting case, a viscous perturbation of the 1-dimensional compressible Euler equations for the ideal polytropic gases. This leads to an improved stability result over the one given in [13].

Consider the 1-dimensional compressible Euler equations in Lagrangian coordinates:

$$
\left\{\begin{array}{l}
\partial_{t} v-\partial_{x} u=0 \\
\partial_{t} u+\partial_{x} p=0 \\
\partial_{t} E+\partial_{x}(u p)=0
\end{array} \quad x \in \mathbb{R}^{1}, \quad t>0\right.
$$

where $v, u, E$, and $p$ are specific volume, velocity, total energy, and pressure respectively. Let $e$ be the internal energy. Then $E=e+\frac{1}{2} u^{2}$. For the ideal polytropic gases [2], the equation of state is given by

$$
p(v, e)=C \frac{e}{v}
$$

with $C$ a positive constant which will be assumed to be one without loss of generality. A contact discontinuity solution to $(4.1)$ can be taken as $(\widetilde{u}, \widetilde{v}, \widetilde{E})$ such that

$$
\widetilde{u} \equiv 0, \quad \widetilde{p} \equiv 1, \quad \widetilde{v}(x, t)= \begin{cases}v_{-}, & x<0 \\ v_{+}, & x>0\end{cases}
$$

where $v_{ \pm}$are positive constants, and $v_{+} \neq v_{-}$.

We study the large time asymptotic behavior of solutions toward the inviscid discontinuity given in (4.3) for the corresponding viscous system of the form

$$
\begin{aligned}
& \left\{\begin{array}{l}
\partial_{t} v-\partial_{x} u=\varepsilon \partial_{x}^{2} v, \\
\partial_{t} u+\partial_{x} p=\varepsilon \partial_{x}^{2} u, \\
\partial_{t} E+\partial_{x}(u p)=\varepsilon \partial_{x}^{2} E,
\end{array} \quad x \in \mathbb{R}^{1}, \quad t>0\right. \\
& (v, u, E)(x, 0)=\left(v_{0}(x), v_{0}(x), E_{0}(x)\right), \quad x \in \mathbb{R}^{1}
\end{aligned}
$$

where $\varepsilon$ is a positive constant. The viscous contact wave $(\bar{v}, \bar{u}, \bar{E})(x, t)$, defined in (2.2), simply becomes

$$
\bar{u}(x, t) \equiv 0, \quad p(\bar{v}, \bar{e})=\bar{p} \equiv 1, \quad\left\{\begin{array}{l}
\partial_{t} \bar{v}=\varepsilon \partial_{x}^{2} \bar{v} \\
\bar{v}(x, t)=v_{-}+\left(v_{+}-v_{-}\right) H(x)
\end{array}\right.
$$

To show that $(\bar{v}, \bar{u}, \bar{E})(x, t)$ is nonlinearly stable, we need only to verify that the structural condition (1.8) are fulfilled in this case. This can be checked easily. Indeed, the Jacobi matrix of the flux function in (4.1) is

$$
A=\left(\begin{array}{rrr}
0 & -1 & 0 \\
-\frac{p}{v} & -\frac{u}{v} & \frac{1}{v} \\
-\frac{u p}{v} & p-\frac{u^{2}}{v} & \frac{u}{v}
\end{array}\right)
$$

The characteristic speeds are eigenvalues of $A$,

$$
\lambda_{1}=-\sqrt{\frac{2 p}{v}}, \quad \lambda_{2} \equiv 0, \quad \lambda_{3}=\sqrt{\frac{2 p}{v}} .
$$


With corresponding right and left eigenvectors

$$
\left\{\begin{array}{l}
r_{1}=\left(\begin{array}{c}
-1 \\
\lambda_{1} \\
p+u \lambda_{1}
\end{array}\right), \quad r_{2}=\left(\begin{array}{l}
1 \\
0 \\
p
\end{array}\right), \quad r_{3}=\left(\begin{array}{c}
-1 \\
\lambda_{3} \\
p+u \lambda_{3}
\end{array}\right), \\
l_{1}=\left(\begin{array}{c}
-\frac{1}{4} \\
-\left(\frac{1}{\sqrt{8}} \sqrt{\frac{v}{p}}+\frac{u}{4 p}\right) \\
\frac{1}{4 p}
\end{array}\right), \quad l_{2}=\left(\begin{array}{c}
\frac{1}{2} \\
\frac{1}{2} u p \\
\frac{1}{2 p}
\end{array}\right), \\
l_{3}=\left(\begin{array}{c}
\frac{1}{4} \\
\frac{1}{\sqrt{8}} \sqrt{\frac{v}{p}}-\frac{u}{4 p} \\
\frac{1}{4 p}
\end{array}\right) .
\end{array}\right.
$$

Note that $\nabla \lambda_{i} \cdot r_{i}=\frac{3}{2} \frac{1}{v} \lambda_{i} \neq 0, i=1,3$, so the corresponding fields are genuinely nonlinear. The waves in these fields are said in the sound wave family. $\lambda_{2} \equiv 0$, so the characteristic field is linearly degenerate, which is called entropy wave family. The inviscid contact discontinuity, defined in (4.3), is associated with this family. For fixed $v_{-}$, the contact wave curve through $\left(v_{-}, u_{-}, E_{-}\right)=\left(v_{-}, 0, v_{-}\right)$is given

$$
v=v_{-}, \quad u \equiv u_{-}=0, \quad p \equiv p_{-}=1 \text {. }
$$

So long the contact curve (4.9), one has

$$
\nabla r_{2} \cdot r_{2}=\left(\begin{array}{c}
0 \\
0 \\
\nabla p \cdot r_{2}
\end{array}\right)=\left(\begin{array}{c}
0 \\
0 \\
\frac{\partial p}{\partial v}+\frac{\partial p}{\partial E} \cdot p
\end{array}\right)=\left(\begin{array}{c}
0 \\
0 \\
-\frac{p}{v}+\frac{p}{v}
\end{array}\right)=\left(\begin{array}{l}
0 \\
0 \\
0
\end{array}\right)
$$

and

$$
\nabla l_{2} \cdot r_{2}=\left(\begin{array}{c}
0 \\
-\frac{1}{2} u \frac{p}{v}+\frac{1}{2} u \frac{p}{v} \\
\frac{1}{2 p v}-\frac{1}{2 p v}
\end{array}\right) \equiv\left(\begin{array}{l}
0 \\
0 \\
0
\end{array}\right)
$$

Consequently, our general theorem in section 2 applies and the leading order asymptotic ansatz is given by a superposition of two nonlinear diffusion waves in the sounds waves families and a shift viscous contact wave in the entropy wave family.

\section{REFERENCES}

[1] I. L. Chern, Multi-molle diffusion waves for viscous nonstrictly hyperbolic conservation laws, Comm. Math. Phys. 138 (1991), pp. 51-61.

[2] R. Courant and K. F, Supersonic Flow and Shock Waves, Wiley-Interscience, New York, 1948.

[3] A. Friedman, Partial Differential Equations of Parabolic Type, Prentice-Hall, Inc., N.J. 1964.

[4] K. Friedrichs and P. D. Lax, Systems of conservation equations with a convex extension, Proc. Nat. Acad. Sci. U.S.A., 68 (1971), pp. 1686-1688.

[5] P. D. Lax, Hyperbolic Systems of Conservation Laws and The Mathematical Theory of Shock Waves, Conf. Board Math. Sci., 11, SIAM, 1973.

[6] T.-P. Liu, Nonlinear Stability of Shock Waves for Viscous Conservation Laws, Memoirs AMS, 56, \#308 (1985).

[7] T.-P. Liu, Pointwise convergence to shock waves for viscous conservation laws, preprint. 
[8] T.-P. Liu and Y. Zeng, Large time behavior of solutions of general quasilinear hyperbolicparabolic systems of conservation laws, preprint, 1995.

[9] T.-P. Liu and Z. P. Xin, Nonlinear stability of rarefaction waves for compressible Navier-Stokes equations, Comm. Math. Physics Vol., 118 (1988), pp. 451-465.

[10] J. Smoller, Shock Waves and Reaction-Diffusion Equations, Springer-Verlag, 1983.

[11] A. Szepessy and Z. P. Xin, Nonlinear stability of viscous shock waves, Arch. Rat. Mech. Anal., 122 (1993), pp. 53-103.

[12] A. Szepessy and K. Zumbrun, Stability of viscous rarefaction waves, preprint.

[13] Z. P. Xin, On nonlinear stability of contact discontinuities, Proceeding of 5th International Conferences on Hyperbolic Problems: Theory, Numerics, and Applications, edited by Glimm, etc. World Scientific, 1996, 249-257. 